\title{
LINEAR DISPERSIVE DECAY ESTIMATES FOR VORTEX SHEETS WITH SURFACE TENSION *
}

\author{
DANIEL SPIRN ${ }^{\dagger}$ AND J. DOUGLAS WRIGHT ${ }^{\ddagger}$
}

\begin{abstract}
We consider the amplitude decay for the linearized equations governing irrotational vortex sheets and water waves with surface tension. Using oscillatory integral estimates, we prove that the magnitude of the amplitude decays faster than $t^{-1 / 3}$.
\end{abstract}

Key words. Water waves, surface tension, vortex sheets, oscillatory integrals, dispersive estimates, Strichartz estimates.

AMS subject classifications. 35Q35, 76B45, 76B47, 76B07, 76B15, 35B45.

\section{Introduction}

Consider a two dimensional, ideal, irrotational fluid with a free interface, denoted by $\Gamma(t)$. The interface is given as the graph of a nonself-intersecting parameterized curve $z(\alpha, t)=(x(\alpha, t), y(\alpha, t))$ where $\alpha \in \mathbf{R}$ is the parameter and $t$ is time. The fluids may shear past one another at the interface but in the bulk of each fluid the flow is assumed to be irrotational. In particular, the vorticity is entirely concentrated on $\Gamma(t)$. Since the velocity is curl-free there is a harmonic potential in the bulk that can be determined if the domain and boundary data along $\Gamma(t)$ is known. Part of the boundary conditions are determined by the kinematic condition in that the interface does not break. This implies the component of the velocity field normal to the surface is continuous across the surface. The remainder of the boundary data is inferred from Euler's equations of motion for inviscid fluids

$$
\mathbf{u}_{t}+(\mathbf{u} \cdot \nabla) \mathbf{u}+\frac{1}{\rho} \nabla p=\mathbf{f}
$$

and the Laplace-Young formula for surface tension

$$
[p]=S \kappa .
$$

Here, $p$ is pressure, $[p]$ is the jump in pressure across $\Gamma, f$ is the sum of body forces (specifically the effects of gravity), $S$ is a constant, $\rho$ is the fluid density and $\kappa$ is the curvature of $\Gamma$. In this way, the equations of motion for the fluid can be posed entirely in terms of quantities evaluated on the free surface, see (A.8). We include a self-contained derivation of the governing equations for this system in Appendix A.

Hou-Lowengrub-Shelley $[15,16]$ developed a highly accurate numerical scheme to study the evolution of $z$ by using, as coordinates, the angle that the surface makes with respect to the horizontal, denoted $\theta(\alpha, t)$, and the jump in tangential velocity across $\Gamma(t)$, denoted $\gamma(\alpha, t)$. Their choice of coordinates was motivated by a desire to reduce the stiffness of numerical simulations. While the curvature $\kappa$ is a nonlinear

* September 25, 2008; accepted (in revised version): March 30, 2009. Communicated by Paul Milewski.

D. Spirn was supported in part by NSF grant DMS-0510121 and DMS-0707714.

J. Wright was supported in part by NSF grant DMS-0807738.

†School of Mathematics, University of Minnesota, Minneapolis, MN 55455, USA (spirn@math. umn.edu).

${ }^{\ddagger}$ Department of Mathematics, Drexel University, Philadelphia, PA 19104, USA (jdoug@math. drexel.edu). 
function of $z_{\alpha}$, it is proportional to $\theta_{\alpha}$. These coordinates are also well-suited for rigorous analysis, see for example $[2,3,5,13]$.

Since the vortex sheet equations (A.8) are nonlinear at the highest order, wellposedness theory poses difficulties; however, local-in-time existence for water waves without surface tension was established by $\mathrm{Wu}[30]$. When surface tension is added, the system gains increased regularity and local well-posedness can be establishedIguchi [17] does so via a graph method and Ambrose [2] uses a more Lagrangian approach. Both authors employ an energy method, and their proofs take particular advantage of structure that is apparent at the linear level. Building on the methods of Ambrose, Ambrose-Masmoudi [3], and Guo-Hallstrom-Spirn [13] improved the energy bounds to the form:

$$
\dot{E} \leq C E(1+E)^{k}
$$

for suitably defined $E$ and a fixed value $k$. However, these bounds are far from implying small data global-in-time existence. Hence, establishing nonlinear stability of two dimensional vortex sheets remains elusive. In certain physical situations, such as when a heavier fluid is placed above a lighter fluid or when there is a large amount of shear, the flat equilibrium state is not stable. Linear instability in these cases has been studied by Hou-Lowengrub-Shelley $[15,16]$ and nonlinear instability was established by Guo-Hallstrom-Spirn [13]. In higher dimensions there have been localin-time results due to Ambrose-Masmoudi [3] on local well-posedness of irrotational vortex sheets and to Cheng-Coutand-Shkoller [8] on local well-posedness of vortex sheets with vorticity in the interior of the fluids.

It is natural to ask whether there exist small-data global-in-time solutions. Typically global existence for nonlinear dispersive equations requires Strichartz estimates in a critical interpolation space and some form of local smoothing. This methodology is well understood for many equations which are closely related to vortex sheets, such as the Korteweg-de Vries (KdV) and nonlinear Schrödinger (NLS) equationssee Bourgain [7], Constantin-Saut [10], Kato [18], Kenig-Ponce-Vega [19], and Tao [27]. Such estimates arise from a conserved energy and a dispersive decay estimate on the linearized equation, so a starting point would be to rigorously establish dispersive estimates for the linearized vortex sheet problem. In particular, one would like to show solutions of the linearized equations decay to zero at an algebraic rate as time evolves, which is the main goal of this paper.

When the physical situation is one in which a heavy fluid, such as water, is below a more rarefied one, such as air, then there are heuristic arguments which indicate that solutions disperse; see for example Chapter 13 of Whitham [29]. In more general situations there is a complicated interplay between the effects of surface tension and gravity, which tend to cause dispersion, and the shear, which tends to cause roll-up of the surface. Such interactions can be sorted out by means of techniques which arise in the study of oscillatory integrals. We consider dispersive estimates on the linearized equations describing vortex sheets with surface tension and gravity in two dimensions.

1.1. Linear dispersive estimates. The vortex sheet system is in equilibrium when the two fluids shear past one another along a perfectly flat interface, that is, when $\theta(\alpha, t)=0$ and $\gamma(\alpha, t)=\bar{\gamma}$. The equations can be found at (A.8). The 
linearization of the equations of motion about this equilibrium is given by

$$
\begin{aligned}
& \partial_{t} \theta=\frac{1}{2} \mathcal{H}\left(\partial_{\alpha} \gamma\right) \\
& \partial_{t} \gamma=\frac{1}{W} \partial_{\alpha \alpha} \theta+\frac{\bar{\gamma}^{2}}{2} \mathcal{H}\left(\partial_{\alpha} \theta\right)-\frac{A}{2} \bar{\gamma} \partial_{\alpha} \gamma-2 A g \theta
\end{aligned}
$$

see also Siegel [23]. Here $W$ is the Weber number and is inversely proportional to the strength with which surface tension acts. The constant $g$ is the acceleration due to gravity and $A:=\frac{\rho_{2}-\rho_{1}}{\rho_{1}+\rho_{2}}$ where $\rho_{j}$ is the density of fluid $j$. The Hilbert transform $\mathcal{H}$ is given by the singular integral

$$
\mathcal{H} f(\alpha):=\frac{1}{\pi} P . V \cdot \int_{\mathbf{R}} \frac{f\left(\alpha^{\prime}\right)}{\alpha-\alpha^{\prime}} d \alpha^{\prime} .
$$

The main result of this paper establishes linear dispersion for (1.1) in the case when the background flow is quiescent, i.e. $\bar{\gamma}=0$.

Theorem 1.1. Suppose that $A>0, g>0,1 / W>0$, and $\bar{\gamma}=0$. If $\theta(\alpha, t)$ and $\gamma(\alpha, t)$ solve (1.1) with initial data $\theta(\alpha, 0)=\theta_{0}(\alpha)$ and $\gamma(\alpha, 0)=\gamma_{0}(\alpha)$, then for any $1 \leq p \leq \infty$ :

$$
\|\theta(t)\|_{L^{\infty}}+\|\gamma(t)\|_{L^{\infty}} \leq C t^{-1 / 3}\left(\left\|\theta_{0}\right\|_{L^{1} \cap \dot{B}_{p, 1}^{2+\frac{1}{p}}}+\left\|\gamma_{0}\right\|_{L^{1} \cap \dot{B}_{p, 1}^{\frac{1}{2}+\frac{1}{p}}}\right),
$$

for $t \geq 1$. (Here $C>0$ is a nonessential constant.)

REMARK 1.1. Though Theorem 1.1 is specialized to $\bar{\gamma}=0$, we expect the same result for $\bar{\gamma}$ in a range determined by the strength of gravity and surface tension. See the discussion below.

REMARK 1.2. That the inital data is forced to lie in the intersection of $L^{1}$ and some Besov space may seem somewhat unnatural. We are free to choose $p$ however. The most fitting choice is $p=1$ since in this case $B_{1,1}^{s} \subset L^{1}$ and thus we only require $\theta_{0} \in B_{1,1}^{3}$ and $\gamma_{0} \in B_{1,1}^{3 / 2}$. Similarly if we take $p=2$ and note that $H^{s} \subset B_{2,1}^{s}$ then we need $\theta_{0} \in L^{1} \cap H^{5 / 2}$ and $\gamma_{0} \in L^{1} \cap H^{1}$.

1.2. Methodology. The first step towards proving linear dispersive estimates is to compute the explicit solution of Equ. (1.1) by means of the Fourier transform. It is

$$
\begin{aligned}
& \widehat{\theta}(\xi, t)=e^{i c_{1} \xi t}\left[\left(-\frac{i c_{1} \xi \sin (\lambda(\xi) t)}{\lambda(\xi)}+\cos (\lambda(\xi) t)\right) \widehat{\theta}_{0}(\xi)+\frac{|\xi| \sin (\lambda(\xi) t)}{\lambda(\xi)} \widehat{\gamma}_{0}(\xi)\right] \\
& \widehat{\gamma}(\xi, t)=e^{i c_{1} \xi t}\left[\frac{c_{1}^{2}-\lambda^{2}(\xi)}{|\xi| \lambda(\xi)} \sin (\lambda(\xi) t) \widehat{\theta}_{0}(\xi)+\left(-\frac{i c_{1} \xi \sin (\lambda(\xi) t)}{\lambda(\xi)}+\cos (\lambda(\xi) t)\right) \widehat{\gamma}_{0}(\xi)\right]
\end{aligned}
$$

where $c_{1}:=-A \bar{\gamma} / 4$ and

$$
\lambda^{2}(\xi):=\frac{1}{2 W}|\xi|^{3}-\frac{\bar{\gamma}^{2}}{4}\left(1-\frac{A^{2}}{4}\right)|\xi|^{2}+A g|\xi| .
$$

By examining $\lambda(\xi)$, one sees how the interaction of gravity, surface tension, and shear affects growth of solutions (see $[1,23]$ ). For example, in the absence of surface 
tension one has $1 / W=0$. This implies that $\lambda^{2}(\xi)<0$ for $\xi$ sufficiently large and thus $e^{i \lambda(\xi) t}$ grows extremely fast for large frequencies. It is this fact that is responsible for the Kelvin-Helmholtz instability and also the ill-posedness of the full nonlinear problem (see [12]). If solutions of (1.1) are to decay, it is necessary that $\lambda^{2}(\xi)>0$ for all $\xi$. This occurs if and only if the inequality

$$
\frac{1}{8} W \bar{\gamma}^{4}\left(1-\frac{A^{2}}{4}\right)^{2}<4 A g
$$

is met. Equivalently, this tells us that gravitation suppresses low frequency modes in much the same way that surface tension suppresses those at high frequencies.

In order to simplify the appearance of the solution operator in (1.2) and the dispersion relation $\lambda(\xi)$ we make the following change of variables:

$$
\alpha^{\prime}=(2 W A g)^{1 / 2} \alpha, \quad t^{\prime}=2^{1 / 4} W^{1 / 4}(A g)^{3 / 4} t, \quad \gamma^{\prime}=\left(\frac{W}{8 A g}\right)^{1 / 4} \gamma
$$

Doing so, setting $\bar{\gamma}=0$, and dropping the "prime" notation results in the equation

$$
\begin{aligned}
\partial_{t} \theta & =\mathcal{H} \partial_{\alpha} \gamma \\
\partial_{t} \gamma & =\partial_{\alpha}^{2} \theta-\theta .
\end{aligned}
$$

The solution of this equation is

$$
\begin{aligned}
& \widehat{\theta}(\xi, t)=\cos (\lambda(\xi) t) \widehat{\theta}_{0}(\xi)+\frac{|\xi| \sin (\lambda(\xi) t)}{\lambda(\xi)} \widehat{\gamma}_{0}(\xi) \\
& \widehat{\gamma}(\xi, t)=-\frac{\lambda(\xi) \sin (\lambda(\xi) t)}{|\xi|} \widehat{\theta}_{0}(\xi)+\cos (\lambda(\xi) t) \widehat{\gamma}_{0}(\xi),
\end{aligned}
$$

with

$$
\lambda(\xi)=\sqrt{|\xi|+|\xi|^{3}} .
$$

If we define the operators

$$
\begin{aligned}
S_{1}(t) f: & :=\mathfrak{F}^{-1}\left[e^{i \lambda(\xi) t} \widehat{f}(\xi)\right] \\
S_{2}(t) f & :=\mathfrak{F}^{-1}\left[e^{i \lambda(\xi) t} \frac{|\xi|}{\lambda(\xi)} \widehat{f}(\xi)\right] \\
S_{3}(t) f: & :=\mathfrak{F}^{-1}\left[e^{i \lambda(\xi) t} \frac{\lambda(\xi)}{|\xi|} \widehat{f}(\xi)\right],
\end{aligned}
$$

then Theorem 1.1 is an immediate consequence of

THEOREM 1.2. There exists $t_{0} \geq 0$ such that for all $t \geq t_{0}$

$$
\begin{gathered}
\left\|S_{1}(t) f\right\|_{L^{\infty}} \leq \frac{C}{t^{1 / 3}}\|f\|_{L^{1} \cap \dot{B}_{p, 1}^{\frac{1}{2}+\frac{1}{p}}} \\
\left\|S_{2}(t) f\right\|_{L^{\infty}} \leq \frac{C}{t^{1 / 3}}\|f\|_{L^{1}}
\end{gathered}
$$


and

$$
\left\|S_{3}(t) f\right\|_{L^{\infty}} \leq \frac{C}{t^{1 / 3}}\|f\|_{L^{1} \cap \dot{B}_{p, 1}^{2+\frac{1}{p}}} .
$$

(Here $p$ is any number in $[1, \infty]$ and $C$ is a nonessential constant.)

REMARK 1.3. The rate of decay of $t^{-1 / 3}$ is the (formally) optimal rate of decay for our system. Notice that for any situation in which (1.4) is met, $\lambda^{\prime}(\xi) \rightarrow \infty$ as $\xi \rightarrow 0^{+}$ and also as $\xi \rightarrow+\infty$. This implies the existence of a point at which $\lambda^{\prime \prime}(\xi)=0$. (This is also connected to the experimentally observed [26] existence of a "slowest wave" in the presence of surface tension.) Thus this problem is more akin to the Airy equation (where the dispersion relation is $\xi^{3}$ and has a decay rate of $t^{-1 / 3}$ ) than the Schrödinger equation (where the dispersion relation is $\xi^{2}$ and has a decay rate of $t^{-1 / 2}$ ). If either surface tension or gravitation is left out, then the decay rate is $t^{-1 / 2}$ - see section 3 . Finally, in [24] we prove that for three-dimensional fluids the analogous dispersive decay rate is $t^{-5 / 6}$.

We divide our proof into three separate sections that handle $S_{1}$ to $S_{3}$ in order. Our primary method is to use oscillatory integral estimates, such as those of Constantin [9] or Kenig-Ponce-Vega [19]. Such tools are called Van der Corput estimates, see for example Stein [25], and they control integrals of highly oscillatory functions by the size of high derivatives of the phase function. In our case the phase function has a more unusual structure than those found in $[6,19]$, therefore we need to develop slightly more general methods.

The oscillatory integrals that we need to control are generally of the form

$$
\int_{a}^{b} \frac{e^{i h(\xi, \kappa)}}{g(\xi)} d \xi
$$

Here $h(\xi, \kappa)$ is the phase and $g$ is an additional factor. This is an example of an oscillatory integral of the second kind and the goal will be to estimate it independently of the parameter $\kappa$. The theory for controlling such integrals is well-developed, but the methods typically require $1 / g(\xi)$ to have compact support (see $[14,25,28]$ ). This is not the case here and in our application there is the further complication that the function $1 / g(\xi)$ may diverge to $\infty$ near the origin or alternately as $|\xi| \rightarrow \infty$. As such, it is extremely important that we keep track of the interplay between the growth of $1 / g(\xi)$ and the delicate cancellations due to the complex exponential. We prove a generalization of the Van der Corput estimate (Lemma 2.2 below) which allows us to control (1.8) by the size of $g h^{\prime}$ (the modified phase) and its derivatives. The proof of this lemma can be found in Appendix B.

Unlike more conventional phase functions, the Van der Corput lemmas are not sufficient to finish the oscillatory integral estimates. In general good dispersive estimates are related to large curvature in the phase function, see [19, 25] etc. Unfortunately the curvature of the modified phase for $S_{1}$ and $S_{3}$ goes to zero for large $|\xi|$ and so we truncate the domain of integration so that we exclude the high frequencies where our decay estimates start to fail. This is a strategy similar to one employed in [22] to prove dispersive estimates for wave equations and is the reason why the Besov spaces appear in Theorem 1.1.

We briefly summarize the rest of the paper. Section 2 describes the Van der Corput lemmas that are used throughout the proof of the theorem along with a lemma for controlling the high frequency pieces. Section 3 contains formal derivation 
of the dispersive decay rate in a variety of scenarios. Sec. $4-6$ handle the $L^{\infty}$ estimates on $S_{1}$ through $S_{3}$. Sec. 7 contains the statement and proof of Strichartz estimates based on our dispersive decay estimates. Appendix A has a self-contained derivation of the irrotational vortex sheet equation with surface tension. Finally, Appendix B contains the proofs of the lemmas in Section 2.

1.3. Notations and definitions. In this document we define the Fourier transform of a function $f(\alpha)$ by

$$
\widehat{f}(\xi):=\mathfrak{F}[f](\xi):=\frac{1}{\sqrt{2 \pi}} \int_{\mathbf{R}} e^{-i \alpha \xi} f(\alpha) d \alpha .
$$

The Sobolev space of order $s$ is denoted by $H^{s}$ and we use as its norm $\|f\|_{H^{s}}^{2}:=$ $\int_{\mathbf{R}}\left(1+\xi^{2}\right)^{s}|f(\xi)|^{2} d \xi$. We denote the inverse Fourier tranform of a function $g(\xi)$ by $\check{g}(\alpha)$. The convolution of $f(\alpha)$ and $g(\alpha)$ is

$$
f * g(\alpha):=\int_{R} f\left(\alpha-\alpha^{\prime}\right) g\left(\alpha^{\prime}\right) d \alpha^{\prime} .
$$

We note that $\widehat{f * g}(\xi)=\frac{1}{\sqrt{2 \pi}} \widehat{f}(\xi) \widehat{g}(\xi)$. The Lebesgue spaces $L^{p}$ are equipped with their standard norms, which we denote by $\|\cdot\|_{L^{p}}$. We will occasionally write $H_{\star}^{s}$ or $L_{\star}^{p}$ where $\star$ stands for the independent variable over which the norm is taken.

We also make use of the homogeneous Besov spaces $\dot{B}_{p, q}^{s}$ with norm defined as

$$
\|u\|_{\dot{B}_{p, q}^{s}}:=\left(\sum_{j=-\infty}^{\infty}\left(2^{s j}\left\|u_{j}\right\|_{L^{p}}\right)^{q}\right)^{\frac{1}{q}} .
$$

Here $u_{j}:=\mathfrak{F}^{-1}\left[\varphi_{j}(\xi) u(\xi)\right]=C \check{\varphi}_{j} \star u$ and the functions $\varphi_{j}$ form a partition of unity attached to the dyadic intervals $|\xi| \in\left[2^{j}, 2^{j+1}\right], j \in \mathbf{N}$. (See Shatah-Struwe [22].)

\section{Preliminary estimates}

As we shall demonstrate in the subsequent sections, controlling the operators $S_{j}(t)$ boils down to controlling the following three oscillatory integrals uniformly in $\kappa:$

$$
\begin{gathered}
\int_{t^{-2 / 3}}^{t^{2 / 3}} e^{i t(\kappa \xi+\lambda(\xi))} d \xi \\
\int_{\mathbf{R}} e^{i t(\kappa \xi+\lambda(\xi))} \frac{|\xi|}{\lambda(\xi)} d \xi \\
\int_{t^{-2 / 9}}^{t^{2 / 9}} e^{i t(\kappa \xi+\lambda(\xi))} \frac{\lambda(\xi)}{|\xi|} d \xi .
\end{gathered}
$$

To do so we will employ the Van der Corput Lemma (see [25]), which states:

LEMMA 2.1. Let $h(\xi)$ be $C^{k}$ on $[a, b]$ with $-\infty \leq a<b \leq+\infty$ and $k \geq 2$. Suppose that $h^{(k)}(\xi)$ is either always positive or always negative on $[a, b]$. Then

$$
\left|\int_{a}^{b} e^{i h(\xi)} d \xi\right| \leq C_{k}\left\{\min _{[a, b]}\left|h^{(k)}\right|\right\}^{-1 / k}
$$


where $C_{k}$ is a positive constant which depends only on $k$ (and not on a or b).

In this document we will need the following generalization of this lemma:

Lemma 2.2. a. Let $h(\xi)$ be $C^{k}$ and $g(\xi)$ be $C^{k-1}$ on $[a, b]$ with $-\infty \leq a<b \leq+\infty$ and $k \geq 2$. Suppose that in $[a, b],\left(g h^{\prime}\right)^{(k-1)}$ is either always positive or always negative and $g \neq 0$. Then

$$
\left|\int_{a}^{b} \frac{e^{i h(\xi)}}{g(\xi)} d \xi\right| \leq C_{k}\left\{\min _{[a, b]}\left|\left(g h^{\prime}\right)^{(k-1)}\right|\right\}^{-1 / k}\left\{\min _{[a, b]}|g|\right\}^{-1+1 / k}
$$

where $C_{k}$ is a positive constant which depends only on $k$ (and not on a or b).

b. Additionally, if $\min _{[a, b]}\left\{\left|g h^{\prime}\right|\right\}>0$ and $\left(g h^{\prime}\right)^{\prime}=0$ at a finite number of points in $[a, b]$ we have

$$
\left|\int_{a}^{b} \frac{e^{i h(\xi)}}{g(\xi)} d \xi\right| \leq C_{1}\left\{\min _{[a, b]}\left|g h^{\prime}\right|\right\}^{-1} .
$$

The proof of this lemma is in Appendix B.

Notice that we estimate the oscillatory integrals above on truncated frequency domains. The following lemma will be used to control the operators outside of these regions:

Lemma 2.3. Fix $s \geq 0, s^{\prime} \geq 0, \beta>0$, and $1 \leq p \leq \infty$. Suppose that $\sigma(\xi) \in C^{\infty}$ and $|\sigma(\xi)| \leq C|\xi|^{s^{\prime}}$. Then, for all $t \geq 1$,

$$
\int_{|\xi| \geq t^{\beta}}|\sigma(\xi) \widehat{f}(\xi)| \leq C t^{-s \beta}\|f\|_{\dot{B}_{p, 1}^{s+s^{\prime}+\frac{1}{p}}} .
$$

The proof of this lemma is also in Appendix B.

\section{Formal stationary phase estimates}

In this section we compute at a formal level the expected dispersive decay rate for the operator $S_{1}(t)$ when (1.4) is met in different physical circumstances.

3.1. Purely gravity waves: $g=1,1 / W=0, \bar{\gamma}=0, A=1$. In this case, notice that that $\lambda(\xi)=|\xi|^{1 / 2}$ and thus

$$
S_{1}(t) f(\alpha)=\frac{1}{\sqrt{2 \pi}} \int_{\mathbf{R}} e^{i\left(\alpha \xi+|\xi|^{1 / 2} t\right)} \widehat{f}(\xi) d \xi .
$$

In particular we will have to estimate oscillatory integrals of the form

$$
\int_{a}^{b} e^{i\left(\alpha \xi+|\xi|^{1 / 2} t\right)} d \xi=\int_{a}^{b} e^{i t\left(\kappa \xi+|\xi|^{1 / 2}\right)} d \xi
$$

where $\kappa=\alpha / t$. Notice that

$$
\frac{\partial^{2}}{\partial \xi^{2}}\left(\kappa \xi+|\xi|^{1 / 2}\right)=-\frac{1}{4}|\xi|^{-3 / 2}
$$

and is independent of $\kappa$. Importantly, this function is bounded away from zero on any bounded interval $[a, b]$. Thus we have from Lemma 2.1 (with $k=2$ ) that

$$
\left|\int_{a}^{b} e^{i\left(\alpha \xi+|\xi|^{1 / 2} t\right)} d \xi\right| \leq C\left\{\min _{[a, b]} t|\xi|^{-3 / 2}\right\}^{-1 / 2} \leq C(b) t^{-1 / 2} .
$$


Thus we expect that in the absence of surface tension, the amplitude of the solution should decay like $t^{-1 / 2}$. Note that $C(b)=O\left(b^{-3 / 4}\right)$ and thus the size of the constant is large for high wave numbers - this can be handled via the truncation methods discussed earlier.

3.2. Purely capillary waves: $g=0,1 / W=1, \bar{\gamma}=0, A=1$. Similarly, if we consider waves in which only surface tension acts, we must estimate

$$
\int_{a}^{b} e^{i t\left(\kappa \xi+|\xi|^{3 / 2}\right)} d \xi
$$

We have

$$
\frac{\partial^{2}}{\partial \xi^{2}}\left(\kappa \xi+|\xi|^{3 / 2}\right)=\frac{3}{4}|\xi|^{-1 / 2}
$$

and it is independent of $\kappa$. As before, this function is bounded away from zero on any bounded interval $[a, b]$ with $a>0$. Thus we have by the same reasoning

$$
\left|\int_{a}^{b} e^{i\left(\alpha \xi+|\xi|^{1 / 2} t\right)} d \xi\right| \leq C(b) t^{-1 / 2} .
$$

(The constant $C(b)$ diverges as $b \rightarrow \infty$, but once again this is a complication that can be handled by truncation.)

3.3. General case: $g>0,1 / W>0, \bar{\gamma} \geq 0$, and with (1.4) met. In this situation, the function $\lambda(\xi)$ is more complicated than in the previous two situations. In particular, notice that when $\xi$ is close to 0 that

$$
\lambda^{\prime}(\xi) \sim c_{1}|\xi|^{-1 / 2}
$$

and that as $|\xi| \rightarrow \infty$

$$
\lambda^{\prime}(\xi) \sim c_{2}|\xi|^{1 / 2}
$$

where both constants $c_{1}$ and $c_{2}$ are positive. Since $\lambda^{\prime}(\xi)$ blows up as $\xi \rightarrow 0$ and as $\xi \rightarrow \infty$, there is clearly a point $0<\xi_{\text {slow }}<\infty$ at which $\lambda^{\prime}(\xi)$ achieves a minimum value and at which $\lambda^{\prime \prime}\left(\xi_{\text {slow }}\right)=0$. Thus it is impossible to apply Lemma 2.1 with $k=2$. It also happens that $\lambda^{\prime \prime \prime}\left(\xi_{\text {slow }}\right) \neq 0$ (at least for $\bar{\gamma} \sim 0$ ) and so Lemma 2.1 can used with $k=3$ to achieve an overall time decay of $t^{-1 / 3}$. This is a surprising result: competition between surface tension and gravitation results in dispersive decay which is slower than in the cases in which solely one of these forces is present. This is related to the fact that in experimental studies of small amplitude water waves, the presence of surface tension causes there to be a non-zero lower bound on wave speed - this "slowest speed" is precisely $\lambda^{\prime}\left(\xi_{\text {slow }}\right)$. The remainder of this paper is dedicated to rigorously justifying the dispersive decay in the situation with $\bar{\gamma}=0$.

4. Estimates for $S_{1}(t)$

In this section we will control the operator $S_{1}$. We have

$$
\begin{aligned}
S_{1}(t) f(\alpha) & =\frac{1}{\sqrt{2 \pi}} \int_{\mathbf{R}} e^{i \alpha \xi} e^{i \lambda(\xi) t} \widehat{f}(\xi) d \xi \\
& =\frac{1}{\sqrt{2 \pi}} \int_{\mathbf{R}} e^{i t \Psi(\xi, \kappa)} \widehat{f}(\xi) d \xi,
\end{aligned}
$$


where $\Psi(\xi, \kappa):=\kappa \xi+\lambda(\xi)$ and $\kappa=\alpha / t$. At times we will suppress the dependence of $\Psi$ on $\kappa$ and simply write $\Psi(\xi)$. Similarly, $\Psi^{(k)}$ means $\partial_{\xi}^{k} \Psi$. Our goal is to estimate $S_{1} f$ independently of $\kappa$.

We break the integral into a low frequency and a high frequency piece:

$$
\begin{aligned}
\left|S_{1}(t) f(\alpha)\right| & =\frac{1}{\sqrt{2 \pi}}\left|\int_{-t^{2 / 3}}^{t^{2 / 3}}+\int_{|\xi| \geq t^{2 / 3}} e^{i t \Psi(\xi, \kappa)} \widehat{f}(\xi) d \xi\right| \\
& \leq C\left\|\int_{-t^{2 / 3}}^{t^{2 / 3}} e^{i t \Psi(\xi, \cdot)} d \xi\right\|_{L_{\kappa}^{\infty}}\|f\|_{L^{1}}+C \int_{|\xi| \geq t^{2 / 3}}|\widehat{f}(\xi)| d \xi .
\end{aligned}
$$

We apply Lemma 2.3 with $s=1 / 2, s^{\prime}=0$ and $\beta=2 / 3$ to control the high frequency piece:

$$
\int_{|\xi| \geq t^{2 / 3}}|\widehat{f}(\xi)| d \xi \leq C t^{-1 / 3}\|f\|_{\dot{B}_{p, 1}^{\frac{1}{2}+\frac{1}{p}}} .
$$

This estimate along with the following proposition prove the first estimate in Theorem 1.2 .

Proposition 4.1. Let $\Psi(\xi, \kappa)=\kappa \xi+\lambda(\xi)$ with $\lambda(\xi)$ as above. Then

$$
\left|\int_{-t^{2 / 3}}^{t^{2 / 3}} e^{i t \Psi(\xi, \kappa)} d \xi\right| \leq \frac{C}{t^{1 / 3}}
$$

where $C$ is independent of $\kappa$.

Proof. Notice that $\Psi^{(k)}$ is independent of $\kappa$ if $k \geq 2$. We compute

$$
\Psi^{\prime \prime}(\xi)=\lambda^{\prime \prime}(\xi)=\frac{3 \xi^{4}+6 \xi^{2}-1}{4 \lambda^{3}(\xi)}
$$

and

$$
\Psi^{\prime \prime \prime}(\xi)=\lambda^{\prime \prime \prime}(\xi)=\operatorname{sgn}(\xi) \frac{-3 \xi^{6}-15 \xi^{4}+15 \xi^{2}+3}{8 \lambda^{5}(\xi)} .
$$

Notice that $\Psi^{\prime \prime}(\xi)=0$ at

$$
\pm \xi_{0}:= \pm \sqrt{\sqrt{\frac{4}{3}}-1}
$$

and that $\Psi^{\prime \prime \prime}(\xi)=0$ only at $\xi= \pm 1$. Let us divide the interval over which the integral in (4.1) is evaluated into the union of the following sets:

$$
\begin{gathered}
I_{0}:=[-\delta, \delta] \\
I_{1}:=\left[-\xi_{0}-\varepsilon,-\xi_{0}+\varepsilon\right] \cup\left[\xi_{0}-\varepsilon, \xi_{0}+\varepsilon\right] \text { and } \\
I_{2}:=\left[-t^{2 / 3},-\xi_{0}-\varepsilon\right] \cup\left[-\xi_{0}+\varepsilon,-\delta\right] \cup\left[\delta, \xi_{0}-\varepsilon\right] \cup\left[\xi_{0}+\varepsilon, t^{2 / 3}\right] .
\end{gathered}
$$

Here $\varepsilon$ and $\delta$ are small, positive constants, as yet undetermined. 
We will control $\int_{I_{j}} e^{i t \Psi(\xi)} d \xi$ for each $j=0,1,2$. Trivially we have

$$
\left|\int_{I_{0}} e^{i t \Psi(\xi)} d \xi\right| \leq 2 \delta
$$

The zeros of $\Psi^{\prime \prime}$ lie in $I_{1}$ and by chosing $\varepsilon$ sufficiently small we can guarantee that $\Psi^{\prime \prime \prime} \neq 0$ within this set. Therefore there is a constant $C_{\varepsilon}>0$ (which depends only on $\varepsilon)$ such that

$$
\min _{I_{1}}\left|\Psi^{\prime \prime \prime}(\xi)\right| \geq C_{\varepsilon}
$$

Thus Lemma 2.2 implies

$$
\left|\int_{I_{1}} e^{i t \Psi(\xi)} d \xi\right| \leq C_{3} C_{\varepsilon}^{-1 / 3} t^{-1 / 3}
$$

In $I_{2}$ the second derivative of $\Psi$ is nonzero. So, by Lemma 2.2 we have

$$
\left|\int_{I_{2}} e^{i t \Psi(\xi)} d \xi\right| \leq C_{2} t^{-1 / 2}\left\{\min _{I_{2}}\left|\Psi^{\prime \prime}\right|\right\}^{-1 / 2} .
$$

The minimum of $\left|\Psi^{\prime \prime}\right|$ in $I_{2}$ occurs at an endpoint of one of the intervals in $I_{2}$ or at \pm 1 , the zeros of $\Psi^{\prime \prime \prime}$. Let

$$
m_{1}:=\min \left\{\left|\Psi^{\prime \prime}( \pm 1)\right|,\left|\Psi^{\prime \prime}\left(\xi_{0} \pm \varepsilon\right)\right|,\left|\Psi^{\prime \prime}\left(-\xi_{0} \pm \varepsilon\right)\right|\right\} .
$$

Note that $m_{1}>0$ and depends only on $\varepsilon$.

From the expression for $\Psi^{\prime \prime}$, we see that there are positive constants $c_{1}$ and $c_{0}$ so that $\left|\Psi^{\prime \prime}( \pm \delta)\right| \geq c_{1} \delta^{-3 / 2}$ and $\left|\Psi^{\prime \prime}\left( \pm t^{2 / 3}\right)\right| \geq c_{0}\left(t^{2 / 3}\right)^{-1 / 2}=c_{0} t^{-1 / 3}$. For $\delta$ sufficiently small and $t$ sufficiently large we can conclude that $c_{1} \delta^{-3 / 2} \geq m_{1} \geq c_{0} t^{-1 / 3}$ and so

$$
\min _{I_{2}}\left|\Psi^{\prime \prime}\right| \geq c_{0} t^{-1 / 3}
$$

Therefore

$$
\left|\int_{I_{2}} e^{i t \Psi(\xi)} d \xi\right| \leq C_{2} t^{-1 / 2}\left\{c_{0} t^{-1 / 3}\right\}^{-1 / 2}=C_{2} c_{0}^{-1 / 2} t^{-1 / 3}
$$

All together, we have

$$
\left|\int_{-t^{2 / 3}}^{t^{2 / 3}} e^{i t \Psi(\xi)} d \xi\right| \leq 2 \delta+C_{d} t^{-1 / 3}
$$

where $C_{d}:=\max \left\{C_{2} c_{0}^{-1 / 2}, C_{3} C_{\varepsilon}^{-1 / 3}\right\}$ and $\delta$ is an arbitrary positive number. This immediately implies (6.1) and we are done.

5. Estimates for $S_{2}(t)$

We have

$$
S_{2}(t) f(\alpha)=\frac{1}{\sqrt{2 \pi}} \int_{\mathbf{R}} e^{i t \Psi(\xi, \kappa)} \frac{|\xi|}{\lambda(\xi)} \widehat{f}(\xi) d \xi
$$


with $\Psi(\xi, \kappa)$ as in the previous section. The convolution estimate shows that

$$
\left|S_{2}(t) f(\alpha)\right| \leq C\left\|\int_{\mathbf{R}} e^{i t \Psi(\xi, \cdot)} \frac{|\xi|}{\lambda(\xi)} d \xi\right\|_{L_{\kappa}^{\infty}}\|f\|_{L^{1}} .
$$

The primary result of this section (which implies the second estimate in Theorem 1.2) is

Proposition 5.1. We have

$$
\left|\int_{\mathbf{R}} e^{i t \Psi(\xi, \kappa)} \frac{|\xi|}{\lambda(\xi)} d \xi\right| \leq \frac{C}{t^{1 / 3}}
$$

for all $\kappa \in \mathbf{R}$ and $t \geq t_{0}$.

Proof. Notice that $\Psi(\xi, \kappa)=\Psi(-\xi,-\kappa)$ and so

$$
\left|\int_{\mathbf{R}} e^{i t \Psi(\xi, \kappa)} \frac{|\xi|}{\lambda(\xi)} d \xi\right|=\left|\int_{\mathbf{R}} e^{i t \Psi(\xi,-\kappa)} \frac{|\xi|}{\lambda(\xi)} d \xi\right| \text {. }
$$

Thus we need only concern ourselves with $\kappa \geq 0$.

In order to establish Proposition 5.1, we need to control the integral over $\mathbf{R}$ by means of Lemma 2.2. To do so we need estimates on the modified phase function

$$
\nu(\xi, \kappa):=\frac{\lambda(\xi)}{|\xi|}\left(\kappa+\lambda^{\prime}(\xi)\right)
$$

and its derivatives with respect $\xi$. These are

$$
\begin{aligned}
\nu & =\left(\frac{\lambda}{|\xi|} \Psi^{\prime}\right)=\frac{\lambda}{|\xi|} \kappa+\frac{\lambda \lambda^{\prime}}{|\xi|} \\
\nu^{\prime} & =\left(\frac{\lambda}{|\xi|} \Psi^{\prime}\right)^{\prime}=\left(\frac{\lambda}{|\xi|}\right)^{\prime} \kappa+\left(\frac{\lambda \lambda^{\prime}}{|\xi|}\right)^{\prime} \\
\nu^{\prime \prime} & =\left(\frac{\lambda}{|\xi|} \Psi^{\prime}\right)^{\prime \prime}=\left(\frac{\lambda}{|\xi|}\right)^{\prime \prime} \kappa+\left(\frac{\lambda \lambda^{\prime}}{|\xi|}\right)^{\prime \prime} .
\end{aligned}
$$

Rewriting the modified phase function from above, we can say that

$$
\begin{aligned}
\nu^{\prime}(\xi) & =\left(\frac{\lambda(\xi)}{|\xi|}\right)\left(\kappa+\lambda^{\prime}(\xi)\right) \\
\nu^{\prime}(\xi) & =\left(\frac{\lambda}{|\xi|}\right)^{\prime}\left(\kappa+\frac{\lambda\left(3 \xi^{2}-1\right)}{\xi\left(\xi^{2}-1\right)}\right) \\
\nu^{\prime \prime}(\xi) & =\left(\frac{\lambda}{|\xi|}\right)^{\prime \prime}\left(\kappa+\frac{4 \lambda\left(1+\xi^{2}\right)}{\xi\left(3+6 \xi^{2}-\xi^{4}\right)}\right) .
\end{aligned}
$$

These imply

- If

$$
\kappa \geq K_{+}(\xi):=-\lambda^{\prime}(\xi)+\frac{|\xi|}{2 \lambda}
$$

then $\nu(\xi) \geq 1 / 2$. 


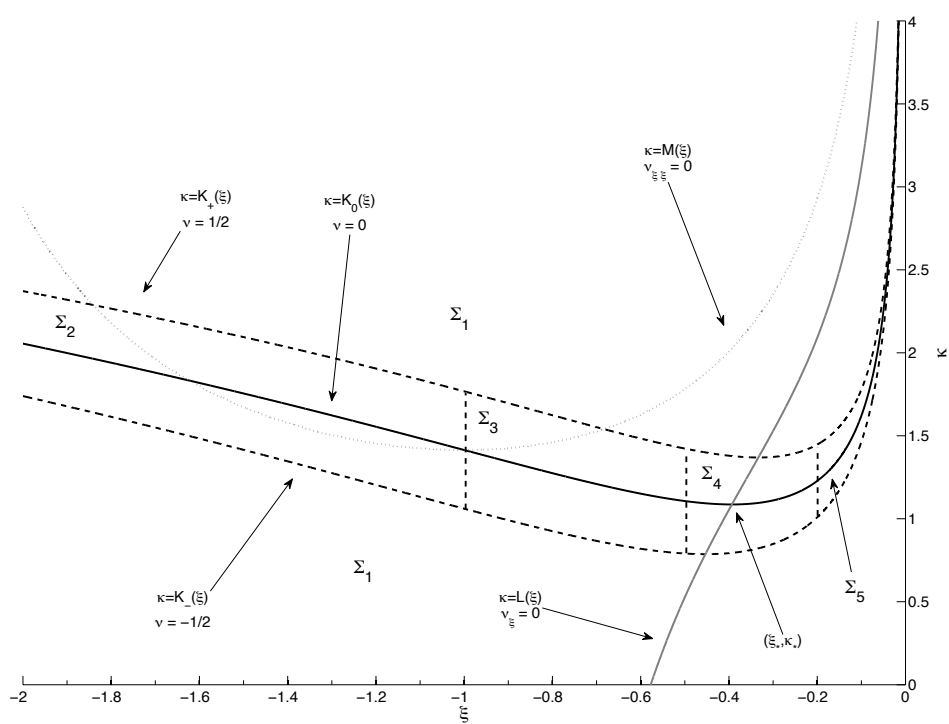

FIG. 5.1. The regions $\Sigma_{j}$ for $S_{2}$.

- If

$$
\kappa \leq K_{-}(\xi):=-\lambda^{\prime}(\xi)-\frac{|\xi|}{2 \lambda},
$$

then $\nu(\xi) \leq-1 / 2$.

- If

$$
\kappa=K_{0}(\xi):=-\lambda^{\prime}(\xi)
$$

then $\nu(\xi)=0$.

Likewise, $\nu_{\xi}(\xi, \kappa)=0$ if and only if

$$
\kappa=L(\xi):=\frac{-\lambda(\xi)\left(3 \xi^{2}-1\right)}{\xi\left(\xi^{2}-1\right)}
$$

and $\nu_{\xi \xi}(\xi, \kappa)=0$ if and only if

$$
\kappa=M(\xi):=\frac{-4 \lambda(\xi)\left(\xi^{2}+1\right)}{\xi\left(3+6 \xi^{2}-\xi^{4}\right)} .
$$

In figure 5.1 we plot the functions $K_{+}, K_{-}, K_{0}, L$, and $M$ when $\xi \leq 0$ and $\kappa \geq 0$. Observe that $\nu$ and $\nu_{\xi}$ are zero simultaneously at one (and only one) point which we denote $\left(\xi_{\star}, \kappa_{\star}\right)$. Note that $-1 / 2 \leq \xi_{\star} \leq-1 / 5$.

Now we define the following sets which cover the upper half of the $\xi \kappa$ plane:

$$
\begin{aligned}
& \Sigma_{0}:=\{(\xi, \kappa) \mid \xi \geq 0 \text { and } \kappa \geq 0\} \\
& \Sigma_{1}:=\left\{(\xi, \kappa) \mid \xi \leq 0 \text { and } \kappa \geq 0 \text { and } \kappa \notin\left(K_{-}(\xi), K_{+}(\xi)\right)\right\} \\
& \Sigma_{2}:=\left\{(\xi, \kappa) \mid \xi \leq-1 \text { and } \kappa \in\left[K_{-}(\xi), K_{+}(\xi)\right]\right\} \\
& \Sigma_{3}:=\left\{(\xi, \kappa) \mid-1 \leq \xi \leq-1 / 2 \text { and } \kappa \in\left[K_{-}(\xi), K_{+}(\xi)\right]\right\} \\
& \Sigma_{4}:=\left\{(\xi, \kappa) \mid-1 / 2 \leq \xi \leq-1 / 5 \text { and } \kappa \in\left[K_{-}(\xi), K_{+}(\xi)\right]\right\}
\end{aligned}
$$


and

$$
\Sigma_{5}:=\left\{(\xi, \kappa) \mid-1 / 5 \leq \xi \leq 0 \text { and } \kappa \in\left[K_{-}(\xi), K_{+}(\xi)\right]\right\} .
$$

We now show that in each of these sets, at least one of $|\nu|,\left|\nu_{\xi}\right|$, or $\left|\nu_{\xi \xi}\right|$ is bounded away from zero.

In $\Sigma_{0}$,

$$
\nu(\xi, \kappa) \geq \frac{\lambda(\xi) \lambda^{\prime}(\xi)}{\xi} \geq \frac{1}{2 \xi}+\frac{3 \xi}{2} \geq \sqrt{3} .
$$

By the definition of $K_{ \pm}(\xi)$ we have

$$
|\nu(\xi, \kappa)| \geq 1 / 2 \text { if }(\xi, \kappa) \in \Sigma_{1} .
$$

There are no zeros of $\nu_{\xi}$ in the set $\Sigma_{3}$ (since the graph of $L$ does not pass through this set). This set is compact and $\nu_{\xi}$ is continuous for all $\xi \neq 0$. Thus we can conclude that there is a constant $c_{3}>0$ such that

$$
\left|\nu_{\xi}(\xi, \kappa)\right| \geq c_{3} \text { if }(\xi, \kappa) \in \Sigma_{3} .
$$

In exactly the same fashion, we see that there are no zeros of $\nu_{\xi \xi}$ in $\Sigma_{4}$ and thus there is a constant $c_{4}>0$ such that

$$
\left|\nu_{\xi \xi}(\xi, \kappa)\right| \geq c_{4} \text { if }(\xi, \kappa) \in \Sigma_{4} .
$$

First we note that in the set $\Sigma_{2}$ and $\Sigma_{5}, \nu_{\xi} \neq 0$. However, these sets are not compact and thus we cannot immediately conclude that $\left|\nu_{\xi}\right|$ is bounded away from zero in these sets. Nevertheless we claim that

$$
\left|\nu_{\xi}(\xi, \kappa)\right| \geq 1 / 8 \text { if }(\xi, \kappa) \in \Sigma_{2}
$$

and

$$
\left|\nu_{\xi}(\xi, \kappa)\right| \geq 1 / 16 \text { if }(\xi, \kappa) \in \Sigma_{5} .
$$

First we cover the case in $\Sigma_{2}$. In this situation, notice that

$$
\nu_{\xi \kappa}=\left(\frac{\lambda(\xi)}{|\xi|}\right)^{\prime}=\frac{\xi^{2}-1}{2 \xi \lambda} \leq 0
$$

for all $(\xi, \kappa) \in \Sigma_{2}$. Thus $\nu_{\xi}$, for fixed a $\xi$, decreases with $\kappa$. This in turn implies that the minimum of $\nu_{\xi}$ must occur on the top boundary of $\Sigma_{2}$ - that is to say, on $\xi=K_{+}(\xi)$. A direct calculation shows

$$
\begin{aligned}
\nu_{\xi}\left(\xi, K_{+}(\xi)\right) & =\left(\frac{\lambda(\xi)}{|\xi|}\right)^{\prime} \frac{\xi}{2 \lambda(\xi)}+\frac{\lambda(\xi) \lambda^{\prime \prime}(\xi)}{|\xi|} \\
& =\frac{(3 \xi+1)\left(-1+2 \xi+\xi^{3}\right)}{4 \xi^{2}\left(1+\xi^{2}\right)} .
\end{aligned}
$$

If $\xi \leq-1$, then $3 \xi+1<\xi<0,-1+2 \xi+\xi^{3}<\xi^{3}<0$, and $\xi^{2}\left(1+\xi^{2}\right) \leq 2 \xi^{4}$ so

$$
\frac{(3 \xi+1)\left(-1+2 \xi+\xi^{3}\right)}{4 \xi^{2}\left(1+\xi^{2}\right)} \geq \frac{(\xi)\left(\xi^{3}\right)}{8 \xi^{4}} \geq \frac{1}{8},
$$


which concludes the validation of (5.6).

In the set $\Sigma_{5}$, we proceed in a similar fashion. In this case, we can conclude that the minimum of $\nu_{\xi}$ must occur on the graph of $K_{-}(\xi)$ since here $\nu_{\xi \kappa}=(\lambda /|\xi|)^{\prime} \geq 0$. Then

$$
\begin{aligned}
\nu_{\xi}\left(\xi, K_{-}(\xi)\right) & =-\left(\frac{\lambda(\xi)}{|\xi|}\right)^{\prime} \frac{\xi}{2 \lambda(\xi)}+\frac{\lambda(\xi) \lambda^{\prime \prime}(\xi)}{|\xi|} \\
& =\frac{(3 \xi-1)\left(1+2 \xi+\xi^{3}\right)}{4 \xi^{2}\left(1+\xi^{2}\right)}
\end{aligned}
$$

For $-1 / 5 \leq \xi \leq 0$, we see $1+2 \xi+\xi^{3} \geq 1-2 / 15-1 / 125 \geq 1 / 2$ and $3 \xi-1 \leq-1$. Thus

$$
\frac{(3 \xi-1)\left(1+2 \xi+\xi^{3}\right)}{4 \xi^{2}\left(1+\xi^{2}\right)} \leq \frac{-1}{8 \xi^{2}\left(1+\xi^{2}\right)} \leq-\frac{1}{16} .
$$

This concludes the validation of (5.7).

Now we can prove Prop. 5.1. Notice that on all of $\mathbf{R} \lambda(\xi) /|\xi|$ is bounded away from zero. Fix $\kappa \geq 0$. If we let

$$
I_{j}(\kappa):=\left\{\xi \mid(\xi, \kappa) \in \Sigma_{j}\right\}
$$

for $j=0, \ldots, 5$, then

$$
\left|\int_{\mathbf{R}} \frac{e^{i t(\kappa \xi+\lambda(\xi))}|\xi|}{\lambda(\xi)} d \xi\right| \leq \sum_{j=0}^{5}\left|\int_{I_{j}(\kappa)} \frac{e^{i t(\kappa \xi+\lambda(\xi))}|\xi|}{\lambda(\xi)} d \xi\right| .
$$

Since $I_{j}(\kappa) \in \Sigma_{j}$, we can use the lower bounds in (5.2) to (5.7) and Lem. 2.2 to conclude that

$$
\sum_{j=0}^{5}\left|\int_{I_{j}(\kappa)} \frac{e^{i t(\kappa \xi+\lambda(\xi))}|\xi|}{\lambda(\xi)} d \xi\right| \leq C\left(t^{-1}+t^{-1 / 2}+t^{-1 / 3}\right) \leq C t^{-1 / 3}
$$

if $t$ is sufficiently large.

6. Estimates for $S_{3}(t)$

In this section we will control the operator $S_{3}$. We have

$$
S_{3}(t) f(\alpha)=\frac{1}{\sqrt{2 \pi}} \int_{\mathbf{R}} e^{i t \Psi(\xi, \kappa)} \frac{\lambda(\xi)}{|\xi|} \widehat{f}(\xi) d \xi,
$$

where $\Psi(\xi, \kappa)$ is as in the previous sections.

We divide the integral into two pieces as was done for $S_{1}(t)$ :

$$
\begin{aligned}
\left|S_{3}(t) f(\alpha)\right| & =\frac{1}{\sqrt{2 \pi}}\left|\int_{-t^{2 / 9}}^{t^{2 / 9}}+\int_{|\xi| \geq t^{2 / 9}} e^{i t \Psi(\xi, \kappa)} \frac{\lambda(\xi)}{|\xi|} \widehat{f}(\xi) d \xi\right| \\
& \leq C\left\|\int_{-t^{2 / 9}}^{t^{2 / 9}} e^{i t \Psi(\xi,)} \frac{\lambda(\xi)}{|\xi|} d \xi\right\|_{L_{\kappa}^{\infty}}\|f\|_{L^{1}}+C \int_{|\xi| \geq t^{2 / 9}}\left|\frac{\lambda(\xi)}{|\xi|} \widehat{f}(\xi)\right| d \xi .
\end{aligned}
$$

For $|\xi| \geq 1, \lambda(\xi) /|\xi| \leq C|\xi|^{1 / 2}$, so we apply Lem. 2.3 with $s=3 / 2, s^{\prime}=1 / 2$, and $\beta=2 / 9$ to control the high frequency piece:

$$
\int_{|\xi| \geq t^{2 / 9}}\left|\frac{\lambda(\xi)}{|\xi|} \widehat{f}(\xi)\right| d \xi \leq C t^{-1 / 3}\|f\|_{\dot{B}_{p, 1}^{2+\frac{1}{p}}} .
$$


This estimate along with the following proposition proves the final estimate in Theorem 1.2.

Proposition 6.1. Let $\Psi(\xi, \kappa)=\kappa \xi+\lambda(\xi)$ with $\lambda(\xi)$ as above. Then

$$
\left|\int_{-t^{2 / 9}}^{t^{2 / 9}} e^{i t \Psi(\xi, \kappa)} \frac{\lambda(\xi)}{|\xi|} d \xi\right| \leq \frac{C}{t^{1 / 3}}
$$

where $C$ is independent of $\kappa$.

Proof.

We need only concern ourselves with $\kappa \geq 0$ for the same reasons as in the previous section. We need to control the integral over $\left[-t^{2 / 9}, t^{2 / 9}\right]$ by means of Lem. 2.2 and thus we need estimates on the modified phase function

$$
\nu(\xi, \kappa):=\frac{|\xi|}{\lambda(\xi)}\left(\kappa+\lambda^{\prime}(\xi)\right)
$$

and its derivatives with respect to $\xi$. Explicitly, $\nu$ and its first two derivatives are

$$
\begin{aligned}
\nu=\left(\frac{|\xi|}{\lambda} \Psi^{\prime}\right) & =\frac{|\xi|}{\lambda} \kappa+\frac{|\xi| \lambda^{\prime}}{\lambda} \\
\nu^{\prime} & =\left(\frac{|\xi|}{\lambda} \Psi^{\prime}\right)^{\prime}=\left(\frac{|\xi|}{\lambda}\right)^{\prime} \kappa+\left(\frac{|\xi| \lambda^{\prime}}{\lambda}\right)^{\prime} \\
\nu^{\prime \prime} & =\left(\frac{|\xi|}{\lambda} \Psi^{\prime}\right)^{\prime \prime}=\left(\frac{|\xi|}{\lambda}\right)^{\prime \prime} \kappa+\left(\frac{|\xi| \lambda^{\prime}}{\lambda}\right)^{\prime \prime} .
\end{aligned}
$$

We can rewrite these as

$$
\begin{aligned}
\nu(\xi) & =\left(\frac{\xi}{\lambda}\right)\left(\kappa+\lambda^{\prime}\right) \\
\nu^{\prime}(\xi) & =\left(\frac{|\xi|}{\lambda}\right)^{\prime}\left(\kappa+\frac{4 \xi \lambda}{1-\xi^{4}}\right) \\
\nu^{\prime \prime}(\xi) & =\left(\frac{|\xi|}{\lambda}\right)^{\prime \prime}\left(\kappa+\frac{8 \operatorname{sgn}(\xi) \xi^{2}\left(3 \xi^{2}-1\right)}{\lambda\left(1+10 \xi^{2}-3 \xi^{4}\right)}\right) .
\end{aligned}
$$

This allows us the make the following observations about $\nu(\xi, \kappa)$.

- If

$$
\kappa \geq K_{+}(\xi):=-\lambda^{\prime}(\xi)+\frac{\lambda(\xi)}{4|\xi|},
$$

then $\nu(\xi) \geq 1 / 4$.

- If

$$
\kappa \leq K_{-}(\xi):=-\lambda^{\prime}(\xi)-\frac{\lambda(\xi)}{4|\xi|}
$$

then $\nu(\xi) \leq-1 / 4$.

- If

$$
\kappa=K_{0}(\xi):=-\lambda^{\prime}(\xi)
$$

then $\nu(\xi)=0$. 


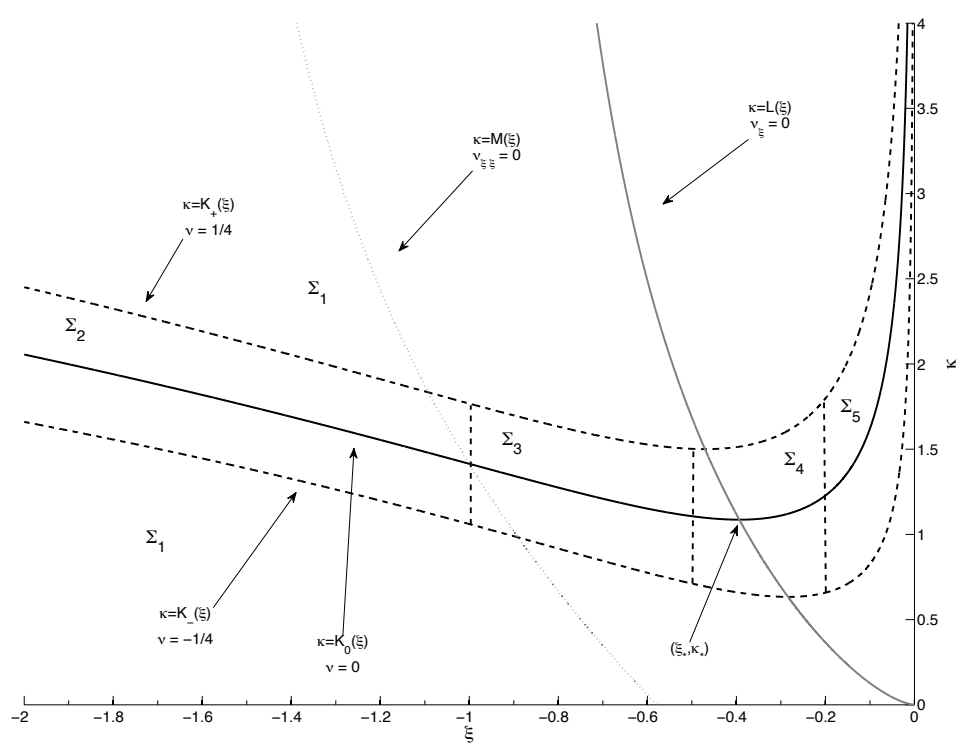

FIG. 6.1. The regions $\Sigma_{j}$ for $S_{3}$.

Similarly $\nu_{\xi}(\xi, \kappa)=0$ if and only if

$$
\kappa=L(\xi):=-\frac{4 \xi \lambda(\xi)}{1-\xi^{4}}
$$

and $\nu_{\xi \xi}(\xi, \kappa)=0$ if and only if

$$
\kappa=M(\xi):=-\frac{8 \operatorname{sgn}(\xi) \xi^{2}\left(3 \xi^{2}-1\right)}{\lambda(\xi)\left(1+10 \xi^{2}-3 \xi^{4}\right)} .
$$

In figure 6.1 we plot the functions $K_{+}, K_{-}, K_{0}, L$, and $M$ when $\xi \leq 0$ and $\kappa \geq 0$. Observe that $\nu$ and $\nu_{\xi}$ are zero simultaneously at one (and only one) point which we denote $\left(\xi_{\star}, \kappa_{\star}\right)$. Note that $-1 / 2 \leq \xi_{\star} \leq-1 / 5$.

Now we define the following sets which cover the region of the $\xi \kappa$ plane where $\kappa \geq 0$ and $|\xi| \leq t^{2 / 9}$ :

$$
\begin{aligned}
& \Sigma_{0}:=\left\{(\xi, \kappa) \mid 0 \leq \xi \leq t^{2 / 9} \text { and } \kappa \geq 0\right\} \\
& \Sigma_{1}:=\left\{(\xi, \kappa) \mid-t^{2 / 9} \leq \xi \leq 0 \text { and } \kappa \geq 0 \text { and } \kappa \notin\left(K_{-}(\xi), K_{+}(\xi)\right)\right\} \\
& \Sigma_{2}:=\left\{(\xi, \kappa) \mid-t^{2 / 9} \leq \xi \leq-1 \text { and } \kappa \in\left[K_{-}(\xi), K_{+}(\xi)\right]\right\} \\
& \Sigma_{3}:=\left\{(\xi, \kappa) \mid-1 \leq \xi \leq-1 / 2 \text { and } \kappa \in\left[K_{-}(\xi), K_{+}(\xi)\right]\right\} \\
& \Sigma_{4}:=\left\{(\xi, \kappa) \mid-1 / 2 \leq \xi \leq-1 / 5 \text { and } \kappa \in\left[K_{-}(\xi), K_{+}(\xi)\right]\right\}
\end{aligned}
$$

and

$$
\Sigma_{5}:=\left\{(\xi, \kappa) \mid-1 / 5 \leq \xi \leq 0 \text { and } \kappa \in\left[K_{-}(\xi), K_{+}(\xi)\right]\right\}
$$


In the sets $\Sigma_{0}, \Sigma_{1}, \Sigma_{2}, \Sigma_{3}, \Sigma_{4}$, at least one of $|\nu|,\left|\nu_{\xi}\right|$ or $\left|\nu_{\xi \xi}\right|$ is bounded away from zero. In the set $\Sigma_{5}$, it is true that $\left|\nu_{\xi}\right|$ is bounded away from zero. Unfortunately, for large values of $\kappa$ this set becomes very close to the $\xi=0$ axis, where $|\xi| / \lambda(\xi)$ is very small. This is problematic when applying Lem. 2.2. We discuss how to deal with $\Sigma_{5}$ in a moment. First we prove lower bounds in the other sets.

In $\Sigma_{0}$,

$$
\nu(\xi, \kappa) \geq \frac{\xi \lambda^{\prime}(\xi)}{\lambda(\xi)}=\frac{3 \xi^{2}+1}{2\left(\xi^{2}+1\right)} \geq 1 / 2 .
$$

By the definition of $K_{ \pm}(\xi)$ we have

$$
|\nu(\xi, \kappa)| \geq 1 / 4 \text { if }(\xi, \kappa) \in \Sigma_{1}
$$

There are no zeros of $\nu_{\xi}$ in the set $\Sigma_{3}$ (since the graph of $L$ does not pass through this set). This set is compact and $\nu_{\xi}$ is continuous for all $\xi \neq 0$. Thus we can conclude that there is a constant $c_{3}>0$ such that

$$
\left|\nu_{\xi}(\xi, \kappa)\right| \geq c_{3} \text { if }(\xi, \kappa) \in \Sigma_{3} .
$$

In exactly the same fashion, we see that there are no zeros of $\nu_{\xi \xi}$ in $\Sigma_{4}$ and thus there is a constant $c_{4}>0$ such that

$$
\left|\nu_{\xi \xi}(\xi, \kappa)\right| \geq c_{4} \text { if }(\xi, \kappa) \in \Sigma_{4}
$$

Note that in the set $\Sigma_{2}, \nu_{\xi} \neq 0$. This set has a boundary which depends on $t$, thus we cannot conclude that $\left|\nu_{\xi}\right|$ is bounded away from zero in a way which is independent of $t$. Still, we claim that

$$
\left|\nu_{\xi}(\xi, \kappa)\right| \geq C t^{-2 / 9} \text { if }(\xi, \kappa) \in \Sigma_{2} .
$$

First we study the interior of $\Sigma_{2}$, and in this case

$$
\nu_{\xi \kappa}=\left(\frac{|\xi|}{\lambda(\xi)}\right)^{\prime}=\frac{\xi\left(1-\xi^{2}\right)}{2 \lambda^{3}(\xi)} \geq 0
$$

for all $(\xi, \kappa) \in \Sigma_{2}$. Thus $\nu_{\xi}$, for fixed a $\xi$, decreases with $\kappa$. This in turn implies that the minimum of $\nu_{\xi}$ must occur on the bottom boundary of $\Sigma_{2}$ - that is to say, on $\xi=K_{-}(\xi)$. A direct calculation shows

$$
\nu_{\xi}\left(\xi, K_{-}(\xi)\right)=-\frac{5 \xi^{4}+12 \xi^{2}-1}{8 \xi\left(1+\xi^{2}\right)^{2}} .
$$

The minimum value of this function occurs at the endpoint $\xi=-t^{2 / 9}$, and thus there exists a constant $C$ such that

$$
\nu_{\xi}\left(\xi, K_{-}(\xi)\right) \geq C t^{-2 / 9}
$$

which concludes the validation of (6.7).

Now we start estimating the oscillatory integral in Prop. 6.1. Fix $\kappa \geq 0$. If we let

$$
I_{j}(\kappa):=\left\{\xi \mid(\xi, \kappa) \in \Sigma_{j}\right\}
$$


for $j=0, \ldots, 5$, then

$$
\left|\int_{\mathbf{R}} \frac{e^{i t(\kappa \xi+\lambda(\xi))}|\xi|}{\lambda(\xi)} d \xi\right| \leq \sum_{j=0}^{5}\left|\int_{I_{j}(\kappa)} \frac{e^{i t(\kappa \xi+\lambda(\xi))}|\xi|}{\lambda(\xi)} d \xi\right| .
$$

Noting that $|\xi| / \lambda(\xi)$ is bounded away from zero in the sets $\Sigma_{3}$ and $\Sigma_{4}$, we apply Lem. 2.2 and conclude using the bounds (6.3), (6.4), (6.5), and (6.6) above that

$$
\left|\int_{I_{0}(\kappa) \cup I_{1}(\kappa) \cup I_{3}(\kappa) \cup I_{4}(\kappa)} \frac{e^{i t(\kappa \xi+\lambda(\xi))}|\xi|}{\lambda(\xi)} d \xi\right| \leq C\left(t^{-1}+t^{-1 / 2}+t^{-1 / 3}\right) \leq C t^{-1 / 3} .
$$

In the set $\Sigma_{2}$, the minimum of $|\xi| / \lambda(\xi)$ occurs at when $\xi=-t^{2 / 9}$. Specifically, we have

$$
\frac{|\xi|}{\lambda(\xi)} \geq C t^{-1 / 9}
$$

Therefore Lem. 2.2 implies that

$$
\left|\int_{I_{2}(\kappa)} \frac{e^{i t(\kappa \xi+\lambda(\xi))}|\xi|}{\lambda(\xi)} d \xi\right| \leq C t^{-1 / 2} t^{1 / 9} t^{1 / 18}=C t^{-1 / 3} .
$$

Finally we estimate the integral in $I_{5}(\kappa)$. First of all, there exists $\kappa_{1}$ such that if $\kappa<\kappa_{1}$ then $I_{5}(\kappa)$ is empty, and there is nothing to estimate. Second of all, we claim that if $\kappa \geq \kappa_{1}$ then

$$
\min _{I_{5}(\kappa)} \frac{|\xi|}{\lambda(\xi)} \geq \frac{C}{\kappa}
$$

and

$$
\min _{I_{5}(\kappa)}\left|\nu_{\xi}(\xi, \kappa)\right| \geq \frac{C}{\kappa^{2}}
$$

If so, then Lem. 2.2 implies that

$$
\left|\int_{I_{5}(\kappa)} \frac{e^{i t(\kappa \xi+\lambda(\xi))}|\xi|}{\lambda(\xi)} d \xi\right| \leq C t^{-1 / 2} \kappa^{-1 / 2} \leq C t^{-1 / 2}
$$

and we would be finished with Prop. 6.1. We now check this claim.

Denote the left and right hand endpoints of $I_{5}(\kappa)$ by $\xi_{1}(\kappa)$ and $\xi_{2}(\kappa)$, respectively. An examination of the functional forms of $K_{ \pm}(\xi)$ shows that there exists $C>1$ such that

$$
\frac{1}{C \kappa^{2}} \leq\left|\xi_{j}(\kappa)\right| \leq \frac{C}{\kappa^{2}}
$$

for $j=1,2$. It is clear that the minimum value of $|\xi| / \lambda(\xi)$ will occur on the right hand endpoint $\xi_{2}(\kappa)$, and this together with the estimates on $\xi_{2}(\kappa)$ confirm (6.10). On the other hand, an examination of the $\nu_{\xi}$ shows that for $0 \geq \xi \geq-1 / 5$ this function is negative and decreasing for any $\kappa$. Thus the minimum of $\left|\nu_{\xi}\right|$ in $I_{5}(\kappa)$ will occur at $\xi_{1}(\kappa)$. The explicit formula for $\nu_{\xi}$ implies (6.11). 


\section{Strichartz estimates}

Given the decay estimates and some natural $L^{2}$ bounds, we can obtain the following class of Strichartz estimates,

Theorem 7.1. For any $\mu \in \mathbb{R}$ we have

$$
\left\|S_{j} g\right\|_{L_{t}^{8}\left(B_{8,2}^{\mu}\right)} \leq C\|g\|_{H^{\mu+\gamma_{j}}},
$$

where $\gamma_{j}=\left\{\frac{9}{16},-\frac{9}{16}, \frac{27}{16}\right\}$, respectively.

We sketch the proof of this result.

Proof. Let $\left\{\widehat{\phi}_{n}\right\}$ be a partition of unity subordinate to the regions

$$
U_{0}=\{|\xi| \in[0,1 / 2]\} \quad U_{n}=\left\{|\xi| \in\left[2^{n-2}, 2^{n-1}\right]\right\}
$$

for $n \geq 1$. Therefore, the support of $\widehat{\phi}_{n}$ is in $U_{n-1} \cup U_{n} \cup U_{n+1}$ if $n \geq 1$ and in $U_{0} \cup U_{1}$ if $n=0$. Let $\chi_{n}(\xi)$ be the characteristic function of $U_{n-1} \cup U_{n} \cup U_{n+1}$ if $n \geq 1$ and the characteristic function of $U_{0} \cup U_{1}$ otherwise. Let $I_{0}:=[0,1 / 2]$ and $I_{n}=\left[2^{n-2}, 2^{n}\right]$ if $n \geq 1$.

In order to prove the Strichartz estimates for the three operators simultaneously, let $\sigma_{j}(s)=\left\{1, \frac{\sqrt{s^{3}+s}}{s}, \frac{s}{\sqrt{s^{3}+s}}\right\}$ and the solution operators are defined as $S_{j} f=$ $\mathcal{F}^{-1}\left(e^{i \lambda(|\xi|) t} \frac{1}{\sigma_{j}(|\xi|)} \hat{f}\right)$. Let $f_{n}=\mathcal{F}^{-1}\left[\chi_{n} \phi_{n} f\right]$, then by a Paley-Littlewood decomposition

$$
\left\|S_{j}(t) f_{n}\right\|_{L^{2}} \leq\left\|\frac{\chi_{n}}{\sigma_{j}} \widehat{\phi}_{n} \widehat{f}\right\|_{L^{2}} \leq \frac{C}{\sigma_{j}\left(2^{n}\right)}\left\|f_{n}\right\|_{L^{2}}
$$

and likewise from Theorem 2 (choosing $p=1$ )

$$
\left\|S_{j}(t) f_{n}\right\|_{L^{1}} \leq C t^{-1 / 3} 2^{s_{j} n}\left\|f_{n}\right\|_{L^{1}}
$$

where $s_{j}=\left\{\frac{3}{2}, 0,3\right\}$. From Riesz-Thorin Interpolation Theorem we find that for $1 \leq$ $r \leq 2$ and $\frac{1}{r}+\frac{1}{q}=1$ then

$$
\left\|S_{j} f_{n}\right\|_{L^{q}} \leq C t^{-\frac{1}{3}\left(\frac{2}{r}-1\right)} 2^{\alpha_{j} n}\left\|f_{n}\right\|_{L^{r}},
$$

where $\alpha_{j}=\left\{\frac{3}{r}-\frac{3}{2}, \frac{1}{r}-1, \frac{5}{r}-2\right\}$.

We use duality to complete the Strichartz estimates. In particular for a test function $\eta$

$$
\left|\left\langle S_{j}(t) g, \eta\right\rangle_{L^{2}\left(\mathbb{R}^{2+1}\right)}\right| \leq\left\|\sigma_{j}^{-1 / 2} \widehat{g}\right\|_{L_{\xi}^{2}}\left\|\int_{t} e^{i t \lambda} \sigma_{j}^{-1 / 2} \bar{\eta} d t\right\|_{L^{2}} .
$$

We estimate the second term on the right hand side. In particular

$$
\left\|\int_{t} e^{i t \lambda} \sigma_{j}^{-1 / 2} \bar{\eta} d t\right\|_{L^{2}} \leq C \sum_{n=0}^{\infty}\left\|\int_{t} e^{i t \lambda} \sigma_{j}^{-1 / 2} \widehat{\eta}_{n} \chi_{n} d t\right\|_{L^{2}}^{2}
$$

and each wavelet is bounded via our dispersive estimate (7.1).

$$
\begin{aligned}
& \left\|\int_{t} e^{i t \lambda} \sigma_{j}^{-1 / 2} \widehat{\eta} \widehat{\phi}_{n} \chi_{n} d t\right\|_{L^{2}}^{2} \\
= & \int_{t} \int_{s}\left(\phi_{n} \star \eta(t), \mathcal{F}^{-1}\left[e^{i(s-t) \lambda} \sigma_{j}^{-1} \chi_{n} \widehat{\phi}_{n} \widehat{\eta}(s)\right]\right)_{L^{2}} d s d t \\
\leq & \int_{t} \int_{s} \frac{2^{\alpha_{j} n}}{|t-s|^{\frac{1}{3}\left(\frac{2}{r}-1\right)}}\left\|\phi_{n} \star \eta(t)\right\|_{L^{r}}\left\|\phi_{n} \star \eta(s)\right\|_{L^{r}} d s d t .
\end{aligned}
$$


Therefore,

$$
\begin{aligned}
&\left\|\int_{t} e^{i t \lambda} \sigma_{j}^{-1 / 2} \widehat{\eta} d t\right\|_{L_{\xi}^{2}}^{2} \\
& \leq C \int_{t} \int_{s}|t-s|^{-\frac{1}{3}\left(\frac{2}{r}-1\right)} \sum_{n=0}^{\infty} 2^{\frac{\alpha_{j} n}{2}}\left\|\phi_{n} \star \eta(t)\right\| 2^{\frac{\alpha_{j} n}{2}}\left\|\phi_{n} \star \eta(s)\right\| d s d t \\
& \leq C \int_{t} \int_{s}|t-s|^{-\frac{1}{3}\left(\frac{2}{r}-1\right)}\|\eta(t)\|_{B_{r, 2}^{\alpha_{j} / 2}}\|\eta(s)\|_{B_{r, 2}^{\alpha_{j} / 2}} \\
& \leq C\|\eta\|_{L^{p}\left(B_{r, 2}^{\alpha_{j} / 2}\right)}^{2}
\end{aligned}
$$

for $p=\frac{2}{2-\frac{1}{3}\left(\frac{2}{r}-1\right)}=\frac{6 r}{7 r-2}$ by the classical Hardy-Sobolev-Littlewood inequality. Choosing $p-r$, then $r=\frac{8}{7}$ and

$$
\left\|\int_{t} e^{i t \lambda} \sigma_{j}^{-1 / 2} \widehat{\eta} d t\right\|_{L_{\xi}^{2}} \leq C\|\eta\|_{L^{8 / 7}\left(B_{8 / 7,2}^{\mu_{j}}\right)}
$$

where $\mu_{j}=\left\{\frac{9}{16},-\frac{1}{16}, \frac{19}{16}\right\}$, respectively. In particular

$$
\left|\left\langle S_{j}(t) g, \eta\right\rangle_{L^{2}\left(\mathbb{R}^{2+1}\right)}\right| \leq C\|g\|_{\dot{H}^{\beta_{j}}}\|\eta\|_{L^{8 / 7}\left(B_{8 / 7,2}^{\mu_{j}}\right)}
$$

with $\beta_{j}=\left\{0,-\frac{1}{2}, \frac{1}{2}\right\}$, so by duality

$$
\left\|S_{j} g\right\|_{L^{8}\left(B_{8,2}^{-\mu_{j}}\right)} \leq C\|g\|_{H^{\beta_{j}}}
$$

If we convolve our initial data $g$ with $(1-\Delta)^{\nu / 2}$ for some real number $\nu=\mu_{j}+\mu$ then

$$
\left\|S_{j} g\right\|_{L^{8}\left(B_{8,2}^{\mu}\right)} \leq C\|g\|_{H^{\mu+\gamma_{j}}}
$$

where $\gamma_{j}=\left\{\frac{9}{16},-\frac{9}{16}, \frac{27}{16}\right\}$.

Appendix A. Derivation \& linearization of equations. We start with a twolayer, inviscid, incompressible, irrotational fluid in $\Omega_{ \pm}$with $\overline{\Omega_{+} \cup \Omega_{-}}=\mathbf{R}^{2}, \Omega \cap \Omega_{-}=\varnothing$ and $\mathbf{R}^{2} \backslash \Omega_{+} \cup \Omega_{-}=\Gamma$, where $\Gamma$ is a smooth, open curve parametrized by $z(\alpha, t)$. Inside each domain we have

$$
\begin{aligned}
\partial_{t} \mathbf{u}+(\mathbf{u} \cdot \nabla) \mathbf{u} & =-\frac{1}{\rho_{ \pm}} \nabla p-g \mathbf{e}_{y} \\
\operatorname{div} u & =0
\end{aligned}
$$

where $g$ is the gravitational constant. Since the fluids are irrotational, we find potential functions $\phi$ and the Bernoulli equations

$$
\partial_{t} \phi+\frac{1}{2}|\nabla \phi|^{2}+\frac{p}{\rho_{ \pm}}+g y=0
$$

inside $\Omega_{ \pm}$. Since $\Delta \phi=0$ in $\Omega_{ \pm}$then there is a double-layer potential and the Plemej Formulae for the velocity of the fluid on either side of the interface

$$
\mathbf{u}_{ \pm}[z(\alpha)]=P V \int K_{2}\left[z(\alpha)-z\left(\alpha^{\prime}\right)\right]\left|z_{\alpha}\right| \gamma\left[z\left(\alpha^{\prime}\right)\right] d \alpha^{\prime} \pm \frac{1}{2} \gamma[z(\alpha)] \mathbf{t}[z(\alpha)]
$$


where $K_{2}[\mathbf{x}]=\frac{\mathbf{x}^{\perp}}{|\mathbf{x}|^{2}}$, see for example [20]. Here $z(\alpha)$ parametrizes $\partial \Omega_{ \pm}$. Set $\mathbf{V}=$ $\frac{1}{2}\left(\mathbf{u}_{+}+\mathbf{u}_{-}\right)$to be the averaged velocity; hence $\frac{1}{2} \nabla\left(\phi_{+}+\phi_{-}\right)=\mathbf{V}$.

We now have two equations

$$
\nabla \phi_{+}+\nabla \phi_{-}=2 \mathbf{V} \quad \text { and } \quad \nabla \phi_{+}-\nabla \phi_{-}=\gamma \mathbf{t}
$$

hence

$$
\nabla \phi_{+}=\mathbf{V}+\frac{1}{2} \gamma \mathbf{t} \quad \text { and } \quad \nabla \phi_{-}=\mathbf{V}-\frac{1}{2} \gamma \mathbf{t}
$$

This implies

$$
\frac{1}{2}\left[\left|\nabla \phi_{+}\right|^{2}+\left|\nabla \phi_{-}\right|^{2}\right]=|\mathbf{V}|^{2}+\frac{1}{4} \gamma^{2} .
$$

We now consider the equation of motion for both $\phi_{+}+\phi_{-}$and $\phi_{+}-\phi_{-}$. We have by the Bernoulli equations:

$$
\begin{aligned}
\partial_{t}\left(\phi_{+}+\phi_{-}\right)+\frac{1}{2}\left[\left|\nabla \phi_{+}\right|+\left|\nabla \phi_{-}\right|^{2}\right]+2 g y & =G=-\frac{p_{+}}{\rho_{+}}-\frac{p_{-}}{\rho_{-}} \\
\partial_{t}\left(\phi_{+}-\phi_{-}\right)+\frac{1}{2}\left[\left|\nabla \phi_{+}\right|-\left|\nabla \phi_{-}\right|^{2}\right]=H & =-\frac{p_{+}}{\rho_{+}}+\frac{p_{-}}{\rho_{-}} .
\end{aligned}
$$

We solve for $p_{+}$and $p_{-}$and obtain

$$
p_{+}=-\frac{\rho_{+}}{2}(H+G) \quad \text { and } \quad p_{-}=\frac{\rho_{-}}{2}(H-G) .
$$

Finally, the Laplace-Young condition implies $p_{-}-p_{+}=S \kappa$, where $\kappa$ is the curvature of the intervace at $z(\alpha)$. Therefore, we obtain

$$
\begin{aligned}
S \kappa & =\frac{\rho_{-}}{2}(H-G)+\frac{\rho_{+}}{2}(H+G) \\
& =\frac{\rho_{+}-\rho_{-}}{2} G+\frac{\rho_{+}+\rho_{-}}{2} H .
\end{aligned}
$$

Finally, we have

$$
H+A G=\frac{1}{W} \kappa
$$

where $A=\frac{\rho_{+}-\rho_{-}}{\rho_{+}+\rho_{-}}$is the Atwood number and $W=\frac{\rho_{+}+\rho_{-}}{2 S}$ is the Weber number.

In order to get the equation of motion for $\gamma$, we differentiate (A.5). Set $z_{\alpha}=$ $\mathbf{t}\left|z_{\alpha}\right|=s_{\alpha} \mathbf{t}$ where $z_{\alpha}=\left(x_{\alpha}, y_{\alpha}\right)$, then $\mathbf{t} \cdot \nabla=s_{\alpha}^{-1}\left(x_{\alpha} \partial_{x}+y_{\alpha} \partial_{y}\right)=s_{\alpha}^{-1} \partial_{\alpha}$. Hence,

$$
\partial_{\alpha}=s_{\alpha} \mathbf{t} \cdot \nabla .
$$

We now differentiate $G$ and $H$ in order to complete the calculation. First

$$
\begin{aligned}
\partial_{\alpha} G & =\partial_{t} \partial_{\alpha}\left(\phi_{+}+\phi_{-}\right)+\partial_{\alpha}\left(|\mathbf{V}|^{2}+\frac{\gamma^{2}}{4}\right)+2 g \partial_{\alpha} y \\
& =\partial_{t}\left[s_{\alpha} \mathbf{t} \cdot \nabla\left(\phi_{+}+\phi_{-}\right)\right]+2 \mathbf{V} \cdot \mathbf{V}_{\alpha}+\frac{\gamma \gamma_{\alpha}}{2}+2 g y_{\alpha} \\
& =2 \partial_{t}\left[s_{\alpha} \mathbf{t} \cdot \mathbf{V}\right]+2 \mathbf{V} \cdot \mathbf{V}_{\alpha}+\frac{\gamma \gamma_{\alpha}}{2}+2 g y_{\alpha}
\end{aligned}
$$


and next

$$
\begin{aligned}
\partial_{\alpha} H & =\partial_{t} \partial_{\alpha}\left(\phi_{+}-\phi_{-}\right)+\partial_{\alpha}\left[\frac{1}{2} \nabla\left(\phi_{+}+\phi_{-}\right) \cdot \nabla\left(\phi_{+}-\phi_{-}\right)\right] \\
& =\partial_{t}\left[s_{\alpha} \mathbf{t} \cdot \nabla\left(\phi_{+}-\phi_{-}\right)\right]+\partial_{\alpha}[\gamma \mathbf{V} \cdot \mathbf{t}] \\
& =\partial_{t}\left[s_{\alpha} \gamma\right]+\partial_{\alpha}[\gamma \mathbf{V} \cdot \mathbf{t}] .
\end{aligned}
$$

Combining these estimates together yields

$$
\partial_{t}\left[s_{\alpha} \gamma\right]+\partial_{\alpha}[\gamma \mathbf{V} \cdot \mathbf{t}]+A\left[2 \partial_{t}\left[s_{\alpha} \mathbf{t} \cdot \mathbf{V}\right]+2 \mathbf{V} \cdot \mathbf{V}_{\alpha}+\frac{\gamma \gamma_{\alpha}}{2}+2 g y_{\alpha}\right]=\frac{1}{W} \kappa_{\alpha}
$$

We complete by including the equation for the motion of the interface. In particular

$$
\partial_{t} z=\mathbf{V}+R \mathbf{t},
$$

where $R$ is an arbitrary tangential velocity.

The small scale decomposition (SSD), introduced in [15] allows us to write the curvature term easily. In particular if $\theta=\arctan \left(y_{\alpha} / x_{\alpha}\right)$ then in complex notation $z_{\alpha}=$ $s_{\alpha} e^{i \theta}$. In complex notation $\mathbf{t}=e^{i \theta}$ and $\mathbf{n}=i e^{i \theta}$. Furthermore, $\partial_{t} z_{\alpha}=\mathbf{V}_{\alpha}+R_{\alpha} \mathbf{t}+R \mathbf{t}_{\alpha}$. But since $\partial_{t} z_{\alpha}=\left(\partial_{t} s_{\alpha}\right) e^{i \theta}+i \theta_{t} s_{\alpha} e^{i \theta}=\left(\partial_{t} s_{\alpha}\right) \mathbf{t}+\left(\partial_{t} \theta\right) s_{\alpha} \mathbf{n}$, then $\mathbf{t}_{\alpha} \cdot \mathbf{t}=0$ and $\mathbf{t}_{\alpha} \cdot \mathbf{n}=$ $\theta_{\alpha}$ imply that

$$
\begin{aligned}
s_{\alpha} \partial_{t} \theta & =\mathbf{n} \cdot \partial_{t} z_{\alpha}=\mathbf{n} \cdot \mathbf{V}_{\alpha}+R \theta_{\alpha} \\
\partial_{t} s_{\alpha} & =\mathbf{t} \cdot \partial_{t} z_{\alpha}=\mathbf{t} \cdot \mathbf{V}_{\alpha}+R_{\alpha} .
\end{aligned}
$$

Finally, we note that curvature is simple in the SSD coordinate system: $\kappa \mathbf{n}=\partial_{\alpha} \mathbf{t}=$ $i \theta_{\alpha} e^{i \theta}=\theta_{\alpha} \mathbf{n}$, and so $\kappa=\theta_{\alpha}$. We get our system

$$
\begin{aligned}
s_{\alpha} \partial_{t} \theta= & \mathbf{n} \cdot \mathbf{V}_{\alpha}+R \theta_{\alpha} \\
s_{\alpha} \partial_{t} \gamma= & \frac{1}{W} \theta_{\alpha \alpha}-\gamma \mathbf{n} \cdot \mathbf{V}_{\alpha}-R \gamma \theta_{\alpha}-\partial_{\alpha}[\gamma \mathbf{V} \cdot \mathbf{t}] \\
& \quad-A\left[2 \partial_{t}\left[s_{\alpha} \mathbf{t} \cdot \mathbf{V}\right]+2 \mathbf{V} \cdot \mathbf{V}_{\alpha}+\frac{\gamma \gamma_{\alpha}}{2}+2 g \sin (\theta)\right] \\
\partial_{t} s_{\alpha}= & \mathbf{t} \cdot \mathbf{V}_{\alpha}+R_{\alpha} .
\end{aligned}
$$

There are steady states when $\theta=0, \gamma=\bar{\gamma}, R=\bar{R}$. Choosing a special $R$ such that $R_{\alpha}=-\mathbf{t} \cdot \mathbf{V}_{\alpha}$, then $\partial_{t} s_{\alpha}=0$, which simplifies the calculations. In particular we set $R(\alpha)=-(\mathbf{t} \cdot \mathbf{V})(\alpha)+\int_{-\infty}^{\alpha} \theta_{\alpha} \mathbf{n} \cdot \mathbf{V} d \alpha^{\prime}$. In [15, 2], $R$ is chosen so that the arclength remains constant in time, i.e., $R=T-(\mathbf{V} \cdot \mathbf{t}) \mathbf{t}$ with $T$ a prescribed function; see for example [2]. Note there is no gravity in the equations of motion considered in $[2,3]$. It is straightforward to linearize (A.8) about the steady solution.

We note that the equations of motion for irrotational vortex sheets with no surface tension was written down by Baker-Meiron-Orszag [4]. 


\section{Appendix B. Assorted proofs.}

Proof. (Lem. 2.2) First we prove b. We have

$$
\begin{aligned}
\left|\int_{a}^{b} \frac{e^{i h(\xi)}}{g(\xi)} d \xi\right| & =\left|\int_{a}^{b} \frac{1}{i g h^{\prime}(\xi)}\left(e^{i h(\xi)}\right)^{\prime} d \xi\right| \\
& \leq\left|\frac{1}{i g h^{\prime}(\xi)} e^{i h(\xi)}\right|_{a}^{b}|+| \int_{a}^{b}\left(\frac{1}{i g h^{\prime}(\xi)}\right)^{\prime} e^{i h(\xi)} d \xi \mid \\
& \leq 2\left\{\min _{[a, b]}\left|g h^{\prime}\right|\right\}^{-1}+\int_{a}^{b}\left|\left(\frac{1}{g h^{\prime}(\xi)}\right)^{\prime}\right| d \xi .
\end{aligned}
$$

By assumption, $\left(g h^{\prime}\right)^{\prime}$ is zero at a finite number of points in $(a, b)$. Denote these points by $\xi_{1}<\xi_{2}<\ldots<\xi_{n}$. Also let $\xi_{0}=: a$ and $\xi_{n+1}=b$. In any interval $\left(\xi_{j}, \xi_{j+1}\right)$ notice that $g h^{\prime}$ is monotonic. Then

$$
\begin{aligned}
\int_{a}^{b}\left|\left(\frac{1}{g h^{\prime}(\xi)}\right)^{\prime}\right| d \xi & =\sum_{j=0}^{n} \int_{\xi_{j}}^{\xi_{j+1}}\left|\left(\frac{1}{g h^{\prime}(\xi)}\right)^{\prime}\right| d \xi \\
& =\sum_{j=0}^{n}\left|\int_{\xi_{j}}^{\xi_{j+1}}\left(\frac{1}{g h^{\prime}(\xi)}\right)^{\prime} d \xi\right| \\
& \leq C n\left\{\min _{[a, b]}\left|g h^{\prime}\right|\right\}^{-1} .
\end{aligned}
$$

Now we prove $\mathbf{a}$.

Special Case: $\min _{[a, b]}|g| \geq 1$. The proof is by induction on $k$. Let $k=2$. The assumptions tell us that $g h^{\prime}$ is monotonic. We assume without loss of generality that $\left(g h^{\prime}\right)^{\prime}$ is positive. Since $g h^{\prime}$ is increasing, it crosses the $\xi$-axis either one time or not at all. We assume that it crosses at the point $c \in(a, b)$. (If the crossing occurs at any endpoint or not at all then things proceed in much the same fashion.) Let $0<\delta<<1$ and note that

$$
\int_{a}^{b} \frac{e^{i h(\xi)}}{g(\xi)} d \xi=\left(\int_{a}^{b}+\int_{c-\delta}^{c+\delta}+\int_{c+\delta}^{b}\right) \frac{e^{i h(\xi)}}{g(\xi)} d \xi .
$$

The integral over the middle interval is bounded by $2 \delta$ as a consequence of our special case assumption. Now let us consider the integral over the right hand interval $R:=$ $(c+\delta, b)$. We have

$$
\begin{aligned}
\int_{R} \frac{e^{i h(\xi)}}{g(\xi)} d \xi & =\int_{R} \frac{1}{i g h^{\prime}(\xi)}\left(e^{i h(\xi)}\right)^{\prime} d \xi \\
& =\left.\frac{1}{i g h^{\prime}(\xi)} e^{i h(\xi)}\right|_{c+\delta} ^{b}-\int_{R}\left(\frac{1}{i g h^{\prime}(\xi)}\right)^{\prime} e^{i h(\xi)} d \xi .
\end{aligned}
$$

Since $g h^{\prime}$ is monotonic, we have the estimate

$$
\min _{R}\left|g h^{\prime}\right| \geq \delta \min _{[a, b]}\left|\left(g h^{\prime}\right)^{\prime}\right|
$$


Thus we can majorize the boundary terms in the above integral by

$$
\frac{2}{\delta \min _{[a, b]}\left|\left(g h^{\prime}\right)^{\prime}\right|} \text {. }
$$

We now must control the integral in (B.2). In what follows, we make use of the fact that $\left(g h^{\prime}\right)^{\prime}$ has a definite sign on $[a, b]$. To wit

$$
\begin{aligned}
\left|\int_{R}\left(\frac{1}{i g h^{\prime}(\xi)}\right)^{\prime} e^{i h(\xi)} d \xi\right| & \leq \int_{R}\left|\left(\frac{1}{g h^{\prime}(\xi)}\right)^{\prime}\right| d \xi \\
& \leq\left|\int_{R}\left(\frac{1}{g h^{\prime}(\xi)}\right)^{\prime} d \xi\right| \\
& \leq\left|\frac{1}{g h^{\prime}(\xi)}\right|_{c+\delta}^{b} \mid \\
& \leq \frac{2}{\delta \min _{[a, b]}\left|\left(g h^{\prime}\right)^{\prime}\right|} .
\end{aligned}
$$

To control the integral from $(a, c-\delta)$ follows exactly the same pattern. All told, we have

$$
\left|\int_{a}^{b} \frac{e^{i h(\xi)}}{g(\xi)} d \xi\right| \leq 2 \delta+\frac{8}{\delta \min _{[a, b]}\left|\left(g h^{\prime}\right)^{\prime}\right|} .
$$

Choosing $\delta=\frac{2}{\sqrt{\min _{[a, b]}\left|\left(g h^{\prime}\right)^{\prime}\right|}}$ yields

$$
\left|\int_{a}^{b} e^{i h(\xi)} d \xi\right| \leq \frac{8}{\sqrt{\min _{[a, b]}\left|\left(g h^{\prime}\right)^{\prime}\right|}}
$$

This conclude the base case $k=2$.

Now assume the result for all $2 \leq n<k$. Without loss of generality, assume that $\left(g h^{\prime}\right)^{(k-1)}(\xi)$ is positive on $[a, b]$. This implies that $\left(g h^{\prime}\right)^{(k-2)}(\xi)$ crosses the $\xi$-axis in at most one point. We assume that this happens at $c \in(a, b)$. (If the crossing occurs at and endpoint or not at all then things proceed in much the same fashion.) Let $0<\delta<<1$ and note that

$$
\int_{a}^{b} \frac{e^{i h(\xi)}}{g(\xi)} d \xi=\left(\int_{a}^{b}+\int_{c-\delta}^{c+\delta}+\int_{c+\delta}^{b}\right) \frac{e^{i h(\xi)}}{g(\xi)} d \xi .
$$

The integral over the middle interval is majorized by $2 \delta$ as a consequence of our special case assumption.

Now let us consider the integral over the right hand interval $R:=(c+\delta, b)$. On $R$, we know that $\left(g h^{\prime}\right)^{(k-2)}$ is positive. By the inductive hypothesis, we can conclude that

$$
\left|\int_{R} \frac{e^{i h(\xi)}}{g(\xi)} d \xi\right| \leq C_{k-1}\left\{\min _{R}\left|\left(g h^{\prime}\right)^{(k-2)}\right|\right\}^{-1 /(k-1)} .
$$

Since $\left(g h^{\prime}\right)^{(k-1)}$ is positive, we have the estimate

$$
\min _{R}\left|\left(g h^{\prime}\right)^{(k-2)}\right| \geq \delta \min _{[a, b]}\left|\left(g h^{\prime}\right)^{(k-1)}\right| .
$$


Thus we have

$$
\left|\int_{R} \frac{e^{i h(\xi)}}{g(\xi)} d \xi\right| \leq C_{k-1}\left\{\delta \min _{[a, b]}\left|\left(g h^{\prime}\right)^{(k-1)}\right|\right\}^{-1 /(k-1)} .
$$

Controlling the integral from $(a, c-\delta)$ follows exactly the same pattern. All told, we have

$$
\left|\int_{a}^{b} \frac{e^{i h(\xi)}}{g(\xi)} d \xi\right| \leq 2 \delta+2 C_{k-1}\left\{\delta \min _{[a, b]}\left|\left(g h^{\prime}\right)^{(k-1)}\right|\right\}^{-1 /(k-1)}
$$

Choosing $\delta=\left\{\min _{[a, b]}\left|\left(g h^{\prime}\right)^{(k-1)}\right|\right\}^{-1 / k}$ yields

$$
\left|\int_{a}^{b} \frac{e^{i h(\xi)}}{g(\xi)} d \xi\right| \leq\left(2+2 C_{k-1}\right)\left\{\min _{[a, b]}\left|\left(g h^{\prime}\right)^{(k-1)}\right|\right\}^{-1 / k}
$$

and this completes the proof of Lem. 2.2 under the special case assumption.

General Case: $\min _{[a, b]}|g|>0$. Let $\tilde{g}:=\frac{g}{\min _{[a, b]}|g|}$. Then $\min _{[a, b]}|\tilde{g}|=1$ and we can use the result from the special case to conclude

$$
\left|\int_{a}^{b} \frac{e^{i h(\xi)}}{\tilde{g}(\xi)} d \xi\right| \leq C_{k}\left\{\min _{[a, b]}\left|\left(\tilde{g} h^{\prime}\right)^{(k-1)}\right|\right\}^{-1 / k} .
$$

Rewriting the above in terms of $g$ instead of $\tilde{g}$ results in estimate (2.2) and this lemma is proven.

Proof. (Lem. 2.3) Fix $p$ and let $q$ be its Hölder dual. First we compute

$$
\begin{aligned}
\int_{|\xi|=2^{j}}^{2^{j+1}}|\sigma(\xi) \widehat{f}(\xi)| & \leq C \int_{|\xi|=2^{j}}^{2^{j+1}}|\xi|^{s^{\prime}}|\widehat{f}(\xi)| \varphi_{j}(\xi) d \xi \\
& =C 2^{j s^{\prime}} \int_{|\xi|=2^{j}}^{2^{j+1}}\left|\widehat{f_{j}}(\xi)\right| d \xi \\
& \leq C 2^{j s^{\prime}}\left\|\widehat{f}_{j}\right\|_{L^{q}}\left(2^{j+1}-2^{j}\right)^{\frac{1}{p}} \\
& \leq C 2^{j\left(\frac{1}{p}+s^{\prime}\right)}\left\|\widehat{f}_{j}\right\|_{L^{q}} .
\end{aligned}
$$

(Here $f_{j}:=\check{\varphi}_{j} \star f$.) The Hausdorff-Young inequality states $\|\widehat{f}\|_{L^{q}} \leq\|f\|_{L^{p}}$ for dual exponents $p, q$. Thus

$$
\int_{|\xi|=2^{j}}^{2^{j+1}}|\sigma(\xi) \hat{f}(\xi)| \leq C 2^{j\left(\frac{1}{p}+s^{\prime}\right)}\left\|f_{j}\right\|_{L^{p}}
$$

Now let $N(t):=\left\lfloor\log _{2}\left(t^{\beta}\right)\right\rfloor$ - the smallest integer less than or equal to $\log _{2}\left(t^{\beta}\right)$. 
Then we have

$$
\begin{aligned}
\int_{|\xi| \geq t^{\beta}}|\sigma(\xi) \hat{f}(\xi)| d \xi & \leq \sum_{j=N(t)}^{\infty} \int_{|\xi|=2^{j}}^{2^{j+1}}|\sigma(\xi) \hat{f}(\xi)| d \xi \\
& \leq C \sum_{j=N(t)}^{\infty} 2^{j\left(\frac{1}{p}+s^{\prime}\right)}\left\|f_{j}\right\|_{L^{p}} \\
& \leq C \sum_{j=N(t)}^{\infty} 2^{-s j} 2^{j\left(\frac{1}{p}+s+s^{\prime}\right)}\left\|f_{j}\right\|_{L^{p}} \\
& \leq C 2^{-s N(t)} \sum_{j=N(t)}^{\infty} 2^{j\left(\frac{1}{p}+s+s^{\prime}\right)}\left\|f_{j}\right\|_{L^{p}} \\
& \leq C t^{-s \beta}\|f\|_{\dot{B}_{p, 1}^{s+s^{\prime}+\frac{1}{p}}}
\end{aligned}
$$

Acknowledgement. The authors wish to thank Steve Shkoller for many interesting and helpful discussions.

\section{REFERENCES}

[1] Z. Alterman, Effect of surface tension on the Kelvin-Helmholtz instability of two rotating fluids, 47(1), 224-227, 1961.

[2] D. Ambrose, Well-posedness of vortex sheets with surface tension, SIAM J. Math. Anal., 35, 221-244, 2003.

[3] D. Ambrose and N. Masmoudi, The zero surface tension limit of two-dimensional water waves, Comm. Pure Appl. Math., 58, 1287-1315, 2005.

[4] G. Baker, D. Meiron and S. Orszag, Generalized vortex methods for free-surface flow problems, J. Fluid Mech., 123, 1982.

[5] J.T. Beale, T. Hou and J. Lowengrub, Growth rates for the linearized motion of fluid interfaces away from equilibrium, Comm. Pure Appl. Math., 46, 1269-1301, 1993.

[6] M. Ben-Artzi and J.C. Saut, Uniform decay estimates for a class of oscillatory integrals and applications, Differential Integral Equations, 12, 137-145, 1999.

[7] J. Bourgain, Global Solutions of Nonlinear Schrdinger Equations, American Mathematical Society Colloquium Publications, American Mathematical Society, Providence, RI, 46, 1999.

[8] C.-H. A. Cheng, D. Coutand and S. Shkoller, On the Motion of Vortex Sheets with Surface Tension in the 3D Euler Equations with Vorticity, Commun. Pure Appl. Math., 61, 17151752, 2008.

[9] P. Constantin, Decay estimates for Schrödinger equations, Commun. Math. Phys., 127, 101$108,1990$.

[10] P. Constantin and J.C. Saut, Local smoothing properties of dispersive equations, J. Amer. Math. Soc., 1, 413-439, 1988.

[11] A. Dé Godefroy, Nonlinear decay and scattering of solutions to a Bretherton equation in several space dimensions, Electron. J. Differential Equations, 141, 17, 2005.

[12] D. Ebin, Ill-posedness of the Rayleigh-Taylor and Helmholtz problems for incompressible fluids, Comm. Part. Differ. Equs., 13(10), 1265-1295, 1988.

[13] Y. Guo, C. Hallstrom and D. Spirn, Dynamics near unstable, interfacial fluids, Commun. Math. Phys., 270, 635-689, 2007.

[14] L. Hörmander, The Analysis of Linear Partial Differential Operators I, Springer-Verlag, New York, 1990.

[15] T. Hou, J. Lowengrub and M. Shelley, Removing the stiffness from interfacial flows with surface tension, J. Comp. Phys., 114, 1994.

[16] T. Hou, J. Lowengrub and M. Shelley, The long-time motion of vortex sheets with surface tension, Phys. Fluids, 9, 1933-1954, 1997. 
[17] T. Iguchi, Well-posedness of the initial value problem for capillary-gravity waves, Funkcial. Ekvac., 44, 219-241, 2001.

[18] T. Kato, On the Korteweg-de Vries equation, Manuscript Math., 29, 89-99, 1979.

[19] C. Kenig, G. Ponce and L. Vega, Oscillatory integrals and regularity of dispersive equations, Indiana Univ. Math. J., 40, 33-69, 1991.

[20] A. Majda and A. Bertozzi, Vorticity and incompressible flow, Cambridge Texts in Applied Mathematics, Cambridge University Press, Cambridge, 27, 2002.

[21] M. Ogawa, and A. Tani, Free boundary problem for an incompressible ideal fluid with surface tension, Math. Models Methods Appl. Sci., 12, 1725-1740, 2002.

[22] J. Shatah and M. Struwe, Geometric Wave Equations, American Mathematical Society, Providence, 1998.

[23] M. Siegel, A study of singularity formation in the Kelvin-Helmholtz instability with surface tension, SIAM J. Appl. Math., 55, 865-891, 1995.

[24] D. Spirn and J.D. Wright, Linear dispersive decay estimates for the $3+1$ dimensional water wave equation with surface tension, Canadian Mathematical Bulletin, accepted, January, 2009.

[25] E.M. Stein, Harmonic analysis: real-variable methods, orthogonality, and oscillatory integrals, Princeton Mathematical Series, Monographs in Harmonic Analysis, III. Princeton University Press, Princeton, NJ, 43, 1993.

[26] J.J. Stoker Water Waves, John Wiley \& Sons Inc., New York, 1992.

[27] T. Tao, Low-regularity global solutions to nonlinear dispersive equations Surveys in analysis and operator theory, Canberra, 19-48, 2001. Proc. Centre Math. Appl. Austral. Nat. Univ., 40, Austral. Nat. Univ., Canberra, 2002.

[28] M. Taylor, Partial Differential Equations, Springer, New York, 1996.

[29] G.B. Whitham, Linear and nonlinear waves. Pure and Applied Mathematics, WileyInterscience, New York, 1974.

[30] S. Wu, Well-posedness in Sobolev spaces of the full water wave problem in 2-D, Invent. Math., 130, 39-72, 1997. 